\title{
Synecological response of desert spring benthic prokaryotes and macroinvertebrates to Sierra Nevada roof pendant-derived calcium
}

\section{Ariel D. Friel}

University of Nevada, Las Vegas

Khaled Pordel

University of Nevada

\section{Zach Meyers}

Purdue University

\section{Cale 0. Seymour}

University of Nevada, Las Vegas

Nicole J. Thomas

University of Nevada, Las Vegas

Fred M. Phillips

New Mexico Tech

Jeffrey R. Knott

California State University

Donald W. Sada

Desert Research Institute

\section{Laura Rademacher}

University of the Pacific

Marty Frisbee

Purdue University

Brian P. Hedlund ( $\nabla$ brian.hedlund@unlv.edu )

University of Nevada

\section{Research Article}

Keywords: Sierra Nevada, desert springs, ecohydrogeology, synecology, holdfast, biofilm, benthic macroinvertebrates, $\mathrm{Ca} 2+/ \mathrm{Na}+$ ratio

Posted Date: February 17th, 2022

DOI: https://doi.org/10.21203/rs.3.rs-1326121/v1 
License: (c) (i) This work is licensed under a Creative Commons Attribution 4.0 International License. Read Full License 


\section{Abstract}

Despite the ecological significance of desert springs, little is known about relationships between spring hydrogeochemistry and ecology, particularly over multiple trophic levels. Here, we surveyed microbial communities (bacteria and archaea) and benthic macroinvertebrate (BMI) communities in springs that recharge through granitoid rocks in Owens Valley, CA, to determine whether subtle geochemical differences imparted by Paleozoic roof pendant weathering in the source area affects spring ecosystems. Relative to other springs, roof pendant-recharged springs were characterized by elevated: (i) $\mathrm{Ca}^{2+} / \mathrm{Na}^{+}$, $\mathrm{Ca}^{2+} / \mathrm{Mg}^{2+}$, and divalent/monovalent cation ratios, (ii) relative abundance of benthic aerobes/facultative anaerobes and holdfast/stalked bacteria, and (iii) abundance and diversity of shredder and collectorgatherer BMIs. These BMI feeding groups graze on biofilm communities and stimulate bacterial degradation of particulate organic matter, consistent with extensive bacteria-BMI connections in cooccurrence networks of these springs. Springs not impacted by roof pendants were instead enriched with anaerobes and chemolithotrophs and low-diversity BMI communities with poor bacteria-BMI network connectivity. We speculate that excess $\mathrm{Ca}^{2+}$ derived from roof pendant weathering plays a key role in biofilm formation on coarse granite substrates, with subsequent synergy between benthic microbial biofilms and diverse BMI shredders/collector-gatherers to degrade allochthonous organic material.

\section{Introduction}

Springs emanate where groundwater encounters geologic features such as faults or contacts that direct groundwater to the surface ${ }^{[1]}$. A combination of factors determines spring hydrogeochemistry, including the geology of the recharge zone/aquifer, source geomorphology, and climate ${ }^{[2]}$. Variable spring hydrogeochemistry, physicochemically distinct microenvironments, and the often-isolated nature of springs contribute to the uniqueness and complexity of these spring-supported ecosystems ${ }^{[2,3]}$. Despite their ecological significance, springs are historically understudied, and the connections between spring hydrogeochemistry and ecology are not well understood. Ecohydrogeology is a new, interdisciplinary approach to spring research and characterization that emphasizes integration of geology, chemistry, and biology $[2,3,4]$, with an ultimate goal of improving management strategies to protect springs from threats such as groundwater extraction and climate change ${ }^{[4,5]}$. These threats are particularly pronounced for desert springs that serve as critical habitat given they are the predominant freshwater features in most desert systems ${ }^{[6,7]}$ where climate change is predicted to decrease the magnitude of mountain-system recharge that supports flow in these springs ${ }^{[8]}$. Although there is a dire need for integrated ecohydrogeological studies of springs, especially within desert spring systems, few studies have explored these ecosystems within that framework.

Bacteria and archaea are important in any ecosystem due to their abundance, diversity, and critical roles in biogeochemical cycles ${ }^{[9,10]}$, yet these communities remain understudied in desert springs. Several studies have focused on bacteria and archaea in desert springs within the Cuatro Cienegas Basin (Coahuila, Mexico) that are supported by oligotrophic geothermal groundwater ${ }^{[11,12,13]}$. These studies 
revealed diverse water and benthic communities comprised of Proteobacteria, Actinobacteria, Bacteroidetes, Chloroflexi, Cyanobacteria, and Planctomycetes, among other phyla ${ }^{[11,12,13]}$. In these systems, extremely low P content limits grazing by benthic macroinvertebrates (BMIs), leading to the development of microbialites with dense matrices of extracellular polymeric substances (EPS ${ }^{[14]}$ and extensive biomass and energy cycling between colocalized photoautotrophic Cyanobacteria and heterotrophic bacteria ${ }^{[12]}$. Contrastingly, studies of other desert surface water systems have linked microbial diversity to groundwater flow, salinity, and $\mathrm{pH}$, but not nutrient chemistry ${ }^{[15,16]}$. This is consistent with the known importance of salinity and $\mathrm{pH}$ in structuring microbial communities across multiple biomes ${ }^{[17,18,19,20]}$. Nevertheless, the effects of these and other physicochemical factors on microbial communities in desert springs, and their hydrogeological controls, are not well understood.

Comparatively more research has focused on macroscopic organisms in springs, including plants, fish, and BMIs. In desert springs, BMI communities comprise both insect taxa such as the aquatic larvae of caddisflies, stoneflies, and true flies, and non-insect taxa such as amphipods, gastropods, ostracods, and mollusks ${ }^{[6,7]}$. A variety of physicochemical parameters are correlated with BMI community richness and composition in springs, including temperature, salinity, dissolved oxygen, $\mathrm{pH}$, and alkalinity ${ }^{[7,21,22,23]}$. In related aquatic ecosystems, such as rivers and streams, additional factors like substrate particle size ${ }^{[24,}$ ${ }^{25]}$ and current velocity ${ }^{[26,27]}$ are also important, as many BMls prefer specific substrate types, which can be controlled by current velocity. Importantly, BMls are central to various ecosystem functions and serve as integral members of the food web in aquatic ecosystems ${ }^{[28]}$.

Biological communities in aquatic ecosystems are influenced by both top-down (e.g., grazing and predation of lower trophic levels) and bottom-up processes (e.g., rate of primary productivity or prey availability) that work together to shape community structure and food web interactions over multiple trophic levels ${ }^{[29]}$. Microorganisms are important members of the food web in aquatic communities, playing critical roles in primary production and all stages of decomposition of detritus ${ }^{[30,31]}$. BMls can graze microbial biomass, consume other BMIs, or facilitate detritus decomposition and nutrient cycling through feeding or burrowing activities ${ }^{[28]}$. As such, individual BMI taxa are assigned to functional feeding groups (FFG) based on their preferred feeding behaviors: (1) shredders, which consume microbially conditioned plant litter and other coarse particulate organic matter (CPOM); (2) collectors (gatherers and filterers), which feed on fine particulate organic matter (FPOM) colonized by microbes; (3) scrapers, which graze on periphyton; and (4) predators, which capture and ingest live prey ${ }^{[32,33]}$. BMIs can also serve as prey for vertebrates, transferring energy and nutrients to higher trophic levels ${ }^{[28]}$. Despite the ecological importance of both microorganisms and BMIs, few studies have explored community ecology and food web interactions over multiple domains in springs within a comparative framework of spring hydrogeochemistry.

Owens Valley is a cold semiarid-to-arid desert valley between the Sierra Nevada and White-Inyo Mountains of California with numerous springs found throughout the valley ${ }^{[34]}$. Spring recharge is predominantly via precipitation in the Sierra Nevada. The dominant rock types in the Sierra Nevada are a 
wide range of Cretaceous granitoid rocks with a patchy distribution of Paleozoic metasedimentary and metavolcanic roof pendants and septa also present ${ }^{[35,36]}$. These roof pendants and septa were originally deposited during the Paleozoic Era as marine sediments, beneath which granitic plutons intruded during the Cretaceous ${ }^{[34,37]}$. They formed the 'roof' of the plutons and were subsequently eroded during the late Cretaceous through the Eocene, leaving only patches of the now-metamorphosed roof pendants. The granitoid rocks have an average weight percent (wt.\%) $\mathrm{CaO}$ and $\mathrm{Na}_{2} \mathrm{O}$ of 3.3 and 3.55 , respectively ${ }^{[36]}$. In contrast, the $\mathrm{CaO}$ concentration in Paleozoic roof pendant rocks such as marble and calc-hornfels is $>40$ wt.\% and 19.0 wt.\%, respectively, with $\mathrm{Na}_{2} \mathrm{O}$ concentrations $0.2-0.9 \mathrm{wt} . \%$ in these same rocks ${ }^{[36]}$.

The objective of our study was to determine whether recharge and rock-water interaction through Sierra Nevada roof pendants affects spring geochemistry and aquatic communities across multiple trophic levels in springs that emerge thousands of meters downslope from the pendants. To address this objective, we collected $\sim 60$ physicochemical parameters, 843,111 microbial $16 S$ rRNA genes, and 2,660 individual BMIs from ten mountain-front springs in the Owens Valley that were partially recharged through the roof pendants or only through granitoid rocks and compared their geochemistry and community ecology.

\section{Materials And Methods}

\section{Study Area and Spring Sites}

The Owens Valley ( 1400 m above mean sea level [masl]) is an endorheic basin in the southwestern Great Basin between the Sierra Nevada (max. elev. 4421 masl) and White-Inyo Mountains (max. elev. 4344 masl). Average precipitation in the Sierra Nevada is $>76 \mathrm{~cm} / \mathrm{yr}$ whereas precipitation in Owens Valley $(13 \mathrm{~cm} / \mathrm{yr})$ and White-Inyo Mountains $(18-36 \mathrm{~cm} / \mathrm{yr})$ illustrate the effect of the Sierra Nevada rain shadow ${ }^{[34]}$. Precipitation and surface runoff from the surrounding mountains collect in the Owens River and flow to Owens Lake (dry since 1927) lakebed playa. Annually, Owens Valley average temperatures range from $4{ }^{\circ} \mathrm{C}$ to $35^{\circ} \mathrm{C}$, which explains its cold-arid to cold desert climatic classification ${ }^{[38]}$.

Ten reference springs were sampled at the foot of the eastern Sierra Nevada in the Owens Valley. These springs are broadly distributed from the northern to southern end of the western side of the valley in order to capture geologically heterogeneous recharge zones. Geochemical and microbial samples were collected during March, 2016, May, 2016 and October, 2017. BMI samples and associated aquatic habitat parameters were collected from all of the springs in a single sampling trip in summer 2017. The slope of the spring emergences was calculated using ArcGIS from 1/3 arc second digital elevation models. Field notes, sampling dates, and geographic coordinates are included in Supplementary Table 1 and Supplementary Table 2.

\section{Field Measurements and Hydrogeochemistry Sampling}


Physicochemical characteristics were recorded for each spring source concurrently with the collection of microbial samples, as outlined previously ${ }^{[39]}$. A YSI Professional Plus Quatro multiparameter probe (YSI, Yellow Springs, $\mathrm{OH}$ ) was used for measuring electrical conductance $\left(E C ; \mu \mathrm{Sm}^{-1}\right.$ ), dissolved oxygen (DO; $\%$ and $\left.\mathrm{mg} \mathrm{L}^{-1}\right), \mathrm{pH}$, and temperature at each spring. Specific conductance (SPC; $\left.\mu \mathrm{S} \mathrm{cm}^{-1}\right)$ was calculated

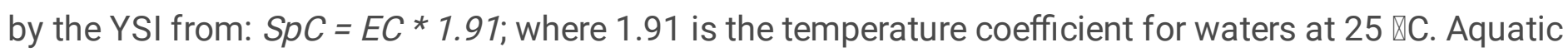
habitat parameters (current velocity, water depth, wetted width, bank cover, head cover, and substrate characteristics) were recorded for each spring while BMI samples were collected. Current velocity was measured using a Marsh-McBirney Flo-Mate 2000 in-stream flow meter (Field Environmental Instruments, INC., Pittsburgh, PA). Both current velocity and water depth represent averaged values of five measurements collected at the center of quadrats where BMls were sampled. Wetted width represents an averaged value of five measurements of width collected along evenly spaced transects oriented perpendicular to the spring channel. Bank cover and head cover were qualitatively estimated. Benthic substrate was characterized in percentages as silt, sand, gravel, cobble, boulder, bedrock, and muck within the BMI sampling area using the Wolman pebble count method. These benthic substrate characteristics represent an averaged value of five measurements of percent substrate present. Quaternary ammonium and $50 \%$ ethanol (95\% ethanol diluted with NanoPure DI water) were utilized on sampling equipment between locations to prevent cross-contamination during sampling trips.

Spring water was collected with a Geopump peristaltic pump (Geotech, Denver, CO) directly from the spring emergence or as close to the source as possible using Masterflex platinum-cured silicone tubing (Cole-Parmer, Vernon Hills, IL) and filtered using $0.2 \mu \mathrm{m}$ polyethersulfone membrane Sterivex-GP pressure filters (Millipore Sigma, Burlington, MA) when relevant. Filtered water samples for measurement of major ions were collected in $250 \mathrm{~mL}$ high-density polyethylene (HDPE) bottles that were rinsed 3 times with NanoPure deionized water (DI) and soaked in DI for a minimum of 24 hours before sampling trips. The bottles were pre-rinsed in the field with fresh spring water 3 times and samples were refrigerated upon collection until analysis. Filtered water samples for trace elements were collected in $60 \mathrm{~mL}$ HDPE bottles. Bottles for trace elements analysis were soaked in 10\% nitric acid (EMD, Omni Trace) for a minimum of 72 hours, rinsed 3 times with NanoPure DI, and dried in a laminar flow hood with a HEPA air filter. $400 \mu \mathrm{L}$ of ultra-pure nitric acid (EMD, Omni Trace Ultra) was added prior to field work to prevent precipitation of metals. Unfiltered water samples were collected for tritium $\left({ }^{3} \mathrm{H}\right)$ and radiocarbon $\left({ }^{14} \mathrm{C}\right)$ analyses, whereas filtered water samples were collected for chlorine-36 $\left({ }^{36} \mathrm{Cl}\right)$ analysis. ${ }^{3} \mathrm{H},{ }^{14} \mathrm{C}$, and ${ }^{36} \mathrm{Cl}$ samples were collected with minimal headspace in $1 \mathrm{~L} \mathrm{HDPE}$ bottles and the caps were sealed with electrical tape to minimize atmospheric exchange.

The major ionic composition of each spring was assessed at the New Mexico Bureau of Geology and Mineral Resources Chemistry Lab. Cations were measured using a PerkinElmer Optima 5300 DV inductively coupled plasma optical emission spectrometry (ICP-OES), according to EPA 200.7. Anions were measured using ion chromatography (IC), according to EPA 300.0. Alkalinity and hardness were measured according to EPA 310.1 and SM 2340B, respectively. Duplicates were run on every tenth sample. Trace elements were measured for most samples at MM Lab (Las Vegas, NV), but the trace 
element sample for one spring (Lubkin Canyon Spring 2 - IES054) was measured at the New Mexico Bureau of Geology and Mineral Resources Chemistry Lab. Trace elements were measured using an Agilent 7500 inductively coupled plasma mass spectrometry (ICP-MS), according to EPA 200.8. Tungsten was measured using an Agilent 7900 ICP-MS system, according to EPA 200.8. ${ }^{3} \mathrm{H}$ samples were analyzed at the Miami Tritium Lab using low-level electrolytic enrichment techniques (https://tritium.rsmas.miami.edu/), ${ }^{14} \mathrm{C}$ analyses were completed by the University of Arizona AMS Laboratory (https://ams.arizona.edu/radiocarbon), and ${ }^{36} \mathrm{Cl}$ analyses were completed at the Purdue PRIME Lab (http://www.physics.purdue.edu/primelab).

\section{Analysis of Spring Hydrogeochemistry Data}

Prior to hierarchical clustering analysis $(\mathrm{HCA})$ or plotting of Piper diagrams, milliequivalents/kilogram (meq/kg) major ion data $\left(\mathrm{Ca}^{2+}, \mathrm{Mg}^{2+}, \mathrm{K}^{+}, \mathrm{Na}^{+}, \mathrm{Cl}^{-}, \mathrm{SO}_{4}{ }^{2-}\right.$, and $\left.\mathrm{HCO}_{3}{ }^{-}\right)$were normalized by dividing each cation by the sum of all the cations in the sample and each anion by the sum of all the anions in the sample to get the final units of \% meq/ $\mathrm{kg}$. Values for specific conductance and Si included in the HCA were normalized prior to the analysis. Specific conductance was log-transformed initially, then both the specific conductance and $\mathrm{Si}$ values were normalized to a value between zero and one using the $\mathrm{R}$ package SuperPCA version $0.3 .0^{[40]}$. To identify the hydrogeochemical groups, an HCA was performed using a Euclidean-based dissimilarity matrix of the transformed data with the R package stats version 4.0.3. Ward's Linkage was used as the linkage type. To identify major spring water types and geochemical differences between the hydrogeochemical groups, Piper diagrams were generated using Geochemist's Workbench Community Edition 15.0 (https://community.gwb.com/). Additionally, a twosample student's t-test was conducted using the R package stats on the physicochemical data (units as shown in Supplementary Table 1) to determine which specific parameters were significantly different between the hydrogeochemical groups. An expanded discussion of the hydrogeochemical framework and analysis is available in another study ${ }^{[41]}$. Major ion or trace element values observed to be below the detection limit $(D L)$ were set to $(D L / 2)$ if they were included in an analysis. Analytes with $>50 \%$ of the concentrations below the DL were excluded from analyses. DLs and analytical uncertainties are included in Supplementary Table 1.

\section{Analysis of Spring Residence Times}

Spring waters were dated using a sequential multi-tracer approach using ${ }^{3} \mathrm{H},{ }^{14} \mathrm{C}$, and ${ }^{36} \mathrm{Cl}$ in that order, as outlined previously ${ }^{[42]}$. First, ${ }^{3} \mathrm{H}$ dating was used to identify the youngest spring waters (e.g., the shortest residence times). Suitable spring water samples with ${ }^{3} \mathrm{H}$ activities $>0.80 \mathrm{TU}$ were dated using TracerLPM ${ }^{[43]}$. A time series of atmospheric ${ }^{3} \mathrm{H}$ from Modesto, $\mathrm{CA}$ was chosen as the input function to the convolution integral since it represents the closest and most complete times series for the study area. A dispersion model was selected as the response function because it more accurately represents mixing of different groundwater flowpaths in spring discharge ${ }^{[43]}$. The lower and upper age constraints in the dispersion model were kept constant at 0 and 400 years, respectively, and the dispersion parameter (DP), 
equal to the inverse of the Peclet number, was initialized to 0.50. Because TracerLPM minimizes error as it seeks a solution within the specified boundaries, the analytical error reported for each water sample by the lab was used to assess numerical uncertainty in the model. The results from TracerLPM were compared to residence times calculated using a recharge-weighted, steady-state, backward-in time tritium mixing mode ${ }^{[42]}$ to identify discrepancies in residence times. The raw data, analytical uncertainty, estimated residence time, and numerical uncertainty associated with ${ }^{3} \mathrm{H}$ dating are reported in

\section{Supplementary Table 1 and Supplementary Table 3.}

Second, ${ }^{14} \mathrm{C}$ dating was used to date spring water samples having ${ }^{3} \mathrm{H}$ activities $<0.80$ TU and ${ }^{14} \mathrm{C} \mathrm{pMC}$ (percent modern carbon) and $\mathrm{d}^{13} \mathrm{C}$ values suitable for ${ }^{14} \mathrm{C}$ corrections. The graphical method described in a previous study ${ }^{[44]}$ was used to gain insight into the geochemical reactions affecting the $\delta^{13} \mathrm{C}$ and ${ }^{14} \mathrm{C}$ pMC measured in the spring water samples and to inform the selection of radiocarbon correction models. ${ }^{14} \mathrm{C}$ corrections were completed using Netpath $\mathrm{XL}^{[45]}$ by 1 ) inputting the measured $\delta^{13} \mathrm{C}$ and ${ }^{14} \mathrm{C}$ pMC of each water sample, 2) specifying the $\delta^{13} \mathrm{C}$ and ${ }^{14} \mathrm{C} \mathrm{pMC}$ of carbonate in soil and bedrock, and $\mathrm{CO}_{2}$ in the soil, and 3 ) testing different ${ }^{14} \mathrm{C}$ correction models ${ }^{[46,47,48,49,50]}$. Hand calculations were performed by allowing the carbonate endmembers to vary substantially and then seeking suitable residence times within the potential solutions. For this step, $\delta^{13} \mathrm{C}_{\text {rec }}$ (the $\delta^{13} \mathrm{C}$ of recharge) was allowed to vary from $0 \%$ to $-22 \%$ o, $\delta^{13} C_{\text {carb }}$ (the $\delta^{13} \mathrm{C}$ of bedrock calcite) was allowed to vary from $0 \%$ o to $-10 \%$. However, the $\delta^{13} \mathrm{C}_{\text {carb }}$ values of roof-pendant marble and disseminated calcite in the granitic plutonic bedrock likely fall within the range of $0 \%$ o to $-5 \%$, but disseminated calcite precipitated in fractures by groundwater could be relatively modern and hence, more negative. The raw data, analytical uncertainty, estimated residence time, and numerical uncertainty associated with ${ }^{14} \mathrm{C}$ dating are reported in Supplementary Table 1 and Supplementary Table 3.

Finally, a chlorine-36 chronometer $\left({ }^{36} \mathrm{Cl} / \mathrm{Cl}\right.$ chronometer) was utilized to estimate the residence times of spring water samples that weren't suitable for either ${ }^{3} \mathrm{H}$ or ${ }^{14} \mathrm{C}$ dating ${ }^{[42]}$. The ${ }^{36} \mathrm{Cl} / \mathrm{Cl}$ chronometer was produced by fitting a trendline between the ${ }^{36} \mathrm{Cl} / \mathrm{Cl}$ ratio and the contributing groundwater residence time of springs in the study that had young residence times (estimated using ${ }^{3} \mathrm{H}$ ) and springs that had old residence times (estimated using ${ }^{14} \mathrm{C}$ ). The power-law trendline $\left(\mathrm{r}^{2}=0.76\right)$ of the chronometer was found to be: $R . T .=700,670 *(36 \mathrm{Cl} / \mathrm{Cl})^{-1.06}$ where; R.T. is residence time in years and ${ }^{36} \mathrm{Cl} / \mathrm{Cl}$ is the chlorine-36 ratio of the spring water sample. An envelope of uncertainty surrounds the chronometer since there is analytical uncertainty in the measured ${ }^{3} \mathrm{H}$ and ${ }^{36} \mathrm{Cl} / \mathrm{Cl}$ compositions of the spring water and numerical uncertainty in the estimation of ${ }^{3} \mathrm{H}$ and ${ }^{14} \mathrm{C}$ residence times. Uncertainty in the ${ }^{36} \mathrm{Cl} / \mathrm{Cl}$ chronometer residence times was determined by linearizing the trendline ${ }^{[42]}$. The raw data, analytical uncertainty, estimated residence time, and numerical uncertainty associated with ${ }^{36} \mathrm{Cl}$ dating are reported in Supplementary Table 1 and Supplementary Table 3.

Organic and Inorganic Nutrient Analysis of Spring Sediment 
One set of the duplicate sediment samples collected for microbial community analysis was utilized to measure the concentration of total organic carbon (TOC), total nitrogen (TN), and total sulfur (TS) in spring sediment. Frozen sediment samples were thawed on ice and once fully thawed were dried at $60{ }^{\circ} \mathrm{C}$ in an incubator until at a constant weight. Dried sediment samples were then ground with a steel mortar and pestle and sieved through an 88-micron sieve. All equipment was rinsed with Barnstead ${ }^{\text {TM }}$ Nanopure $^{\text {TM }}$ water (Thermo Fisher Scientific, Waltham, MA) and dried using Kimtech Science ${ }^{\text {TM }}$ Kimwipes $^{\text {TM }}$ (Kimberley Clark, Irving, TX) in between every sediment sample to clean the tools and prevent nutrient carryover between samples. After processing, sediment samples were sent to the Arizona Laboratory for Emerging Contaminants (ALEC) at the University of Arizona. TOC, TN, and TS concentrations were measured with a Costech ECS-4010 CHNSO analyzer (Costech Analytical Technologies Inc., Valencia, CA), according to ALEC's standard protocol. TOC, TN, and TS values for sediment samples were averaged such that each spring was represented by one TOC, TN, and TS value. TOC, TN, and TS values for each sediment sample are included in Supplementary Table 4.

\section{Microbial Field Sampling}

Water microbial communities were sampled by pumping spring water $(\geq 2 \mathrm{~L}$ total) onto replicate $0.2 \mu \mathrm{m}$ polyethersulfone membrane Sterivex-GP pressure filters (4 filters/spring), as described in detail in the geochemistry 'Field Measurements' section. Excess water was cleared from each filter after sampling with a sterile syringe before sample storage. Benthic microbial biomass was gathered using a sterilized shovel by collecting the top $>2 \mathrm{~cm}$ of spring sediment or benthic microbial mat. Four benthic samples were collected in duplicate at each spring. Sampling locations were selected based on the substrate diversity (e.g., fine-grain sediment, coarse-grain sediment, or microbial mat) of each spring to maximize sampling of unique habitats at every spring. All samples were frozen immediately after sampling and kept frozen on dry ice in the field, and then stored in a $-80{ }^{\circ} \mathrm{C}$ freezer until DNA extraction or organic/inorganic nutrient analysis. Field notes, including descriptions of benthic sample, are detailed in Supplementary Table 2.

\section{Sample Preparation and Sequence Processing for $16 \mathrm{~S}$ rRNA Gene Sequencing}

Microbial DNA was extracted from the samples using the FastDNA ${ }^{\text {TM }}$ SPIN Kit for Soil (MP Biomedicals, Santa Ana, CA) according to the manufacturer's instructions. The V4 region of the 16S rRNA gene was

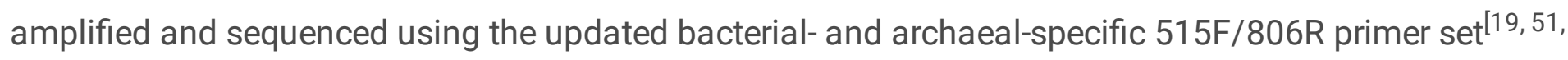
52]. Library prep and paired-end sequencing was conducted at Argonne National Laboratory (Lemont, IL) using an Illumina MiSeq platform (2x151 bp) per the Earth Microbiome Project pipeline (https://earthmicrobiome.org/protocols-and-standards/16s/). Analysis of the 16S rRNA gene sequences was carried out using QIIME2 version 2019.4 ${ }^{[53]}$. Paired-end reads were joined, quality filtered, and assigned to amplicon sequence variants (ASVs) using DADA2 ${ }^{[54]}$. Taxonomy was assigned using a naïveBayesian classifier trained on the V4 region of the Silva NR99 132 alignment ${ }^{[55]}$ via the q2-featureclassifier plugin ${ }^{[56]}$. Sequence variants were aligned using Mafft via the q2-alignment plugin at the 
default settings. Phylogenetic trees were constructed from this alignment using FastTree ${ }^{[57]}$ in QIIME2 version 2019.10. ASVs classified as chloroplast or mitochondria were removed.

\section{BMI Field Sampling and Sample Processing}

BMI communities were sampled no more than $15 \mathrm{~m}$ from each spring source because the source represents the most stable temperature and chemical environment for these communities ${ }^{[58,59]}$. A composite BMI community sample was collected from five subsamples at each spring. Subsamples were collected by placing $120 \mathrm{~cm}^{2}$ quadrats and $10 \mathrm{~cm} \times 12 \mathrm{~cm} 500 \mu \mathrm{m}$ mesh D-frame netting along the same sequence of five equally spaced transects positioned $3 \mathrm{~m}$ apart: center, right bank, center, left bank, and center. Benthic substrate was collected and gently agitated using a quadrat to release BMls from the substrate and allow them to float downstream into the netting. Samples were preserved immediately after collection in the field using $90 \%$ ethyl alcohol. Processing, identification, and enumeration via microscopic examination was conducted at Rhithron Analytical Laboratory (Missoula, MT). A random subsample of at least $300 \mathrm{BMI}$ individuals were identified and enumerated to determine the structure of $\mathrm{BMI}$ communities, this number of individuals has been shown to provide adequate coverage of $\mathrm{BMI}$ community diversity ${ }^{[60]}$. BMI taxa were identified to varying levels of taxonomy. Crenobiotic taxa were identified to the species level, whereas other aquatic insects and mites were identified to the genus level. Triclads (order - Tricladida), oligochaetes (subclass - Oligochaeta), ostracods (class - Ostracoda), and nematodes (phylum - Nematoda) were not identified any further.

\section{a- and $\beta$-Diversity Analysis of Benthic Microbial and Macroinvertebrate Communities}

For microbial samples, rarefaction curves were constructed and plotted with the R package vegan version $2.5-6^{[61]}$. Faith's phylogenetic diversity (Faith's PD) values were calculated for microbial samples using the $\mathrm{R}$ package picante version $1.8^{[62]}$. Three other a-diversity metrics (Observed, Shannon Index, and GiniSimpson Index) were calculated for both microbial and BMI communities using the R packages phyloseq version 1.30 .0 and all metrics were plotted using ggplot2 version 3.3. ${ }^{[63,64]}$. All a-diversity data were tested for normality with the Shapiro-Wilks test using the R package stats. Data that passed the check for normality were then tested for homoscedasticity using the Levene's Test of Equality of Variance (implemented from R package car version 3.0-10) to ensure that the assumption of homogeneity of variance necessary to run ANOVA was not violated ${ }^{[65]}$. If variance was homogenous, data were analyzed with ANOVA and a post-hoc Tukey's Honest Significant Difference Test (where appropriate) using the $\mathrm{R}$ packages stats and agricolae version 1.3-3 ${ }^{[66]}$. Metrics that failed the Shapiro-Wilks test for normality or Levene's Test of Equality of Variance were instead analyzed with a Kruskal-Wallis Test and a post-hoc Dunn Test (where appropriate) using the R packages stats and dunn.test version 1.3.5.

Three approaches were used to explore and compare the patterns in benthic microbial and BMI BrayCurtis community dissimilarity: (1) nonmetric multidimensional scaling (NMDS) analysis, (2) principal coordinate analysis (PCOA), and (3) analysis of similarity (ANOSIM). These analyses were carried out 
using the R packages phyloseq and vegan. Environmental vectors were generated from major ion and trace metals data (in $\mathrm{mol} / \mathrm{L}$ ) and physicochemical parameters via the envfit function of $\mathrm{R}$ package vegan. The Mantel test was used to determine if the benthic microbial and BMI Bray-Curtis community dissimilarity was associated with geographic distance. The distance between springs was calculated using the R package geodist version 0.0.7 ${ }^{[67]}$. Spearman's rank-order correlation Mantel tests (9999 permutations) were conducted on: (1) unaveraged benthic microbial samples, (2) averaged benthic microbial samples, and (3) BMI samples using the package vegan. Prior to the Mantel test, benthic microbial community data were averaged together to control for the presence of zero-distance comparisons when analyzing multiple samples from the same spring.

The taxonomic composition of the water and benthic microbial communities in the two geochemical groups (granitic and roof pendant springs) were assessed independently of each other and independently of the BMI community. Microbial taxa were agglomerated at the phylum, order, and genus level and BMI taxa at the order and genus level. Microbial ASV and BMI count data were then converted to percent relative abundance and a cutoff was applied to merge low abundance taxa. Taxonomic agglomeration and data transformation for the biological communities was carried out using phyloseq and taxonomic barplots were plotted using ggplot2. To identify differentially enriched benthic microbial taxa in granitic and roof pendant springs, a linear discriminant analysis effect size (LEfSe) analysis was conducted using the $\mathrm{R}$ package microbiomeMarker version 0.0.1.9000[68] . As suggested, benthic microbial relative abundance data was normalized using a counts per million (CPM) transformation prior to conducting the LEfSe analysis ${ }^{[68]}$.

Triplicate microbial water samples were originally sequenced for two springs: Grover Anton Spring (IES 028) and Elderberry Canyon Spring (IES 033), as part of an effort to constrain the variability in microbial community structure between replicate water samples. Given the similarity of the triplicate water samples to each other, the microbial ASV counts for those samples were averaged together for each spring individually. These averaged ASV counts were utilized for most analyses, except for the rarefaction curves and Faith's PD analysis, which were run prior to averaging the triplicate water samples. Several plots were generated for the water microbes and included in the supplement with supporting text, however, the water microbial community plots were not analyzed in detail in-text due to the weak relationship observed with the geochemical groups (Supplementary Figures 1-6).

\section{Co-Occurrence Analysis of Microbial and BMI Communities}

The relationship between the relative abundances of benthic microbes and BMls was visualized using a correlation co-occurrence network map. First, benthic microbial and BMI taxa were taxonomically agglomerated at the genus level and converted to percent relative abundance. Taxa representing $<7.5 \%$ (benthic microbes) or $<5 \%$ (BMIs) across the datasets were then merged. Next, Spearman's rank-order correlation coefficients and $p$-values were calculated between the community relative abundance matrices using the R package Hmisc version 4-4.0 ${ }^{[69]}$. Correlation co-occurrence network maps were constructed using the $\mathrm{R}$ package network version $1.16 .1^{[70,71]}$, and then plotted using the $\mathrm{R}$ package 
ggnet version 0.1.0 ${ }^{[72]}$. Genera were color-coded on the maps by BMI stress tolerance/microbial metabolism or BMI behavior/microbial attachment. Stress tolerance values (range: 0-10) were assigned to BMI taxa as previously characterized with intolerant taxa denoted by low stress tolerance values and tolerant taxa denoted by high stress tolerance values ${ }^{[73]}$.

\section{Results}

\section{Paleozoic Roof Pendants Impart Subtle Geochemical Differences in Similar Springs}

The ten springs along the foot of the eastern Sierra Nevada provide a broad geographic and geologic representation of springs along the west side of Owens Valley (Figure 1A). These rheocrene type springs ${ }^{[3]}$ were physicochemically similar, being characterized by cool, fresh water (temperature: 11.2-18.7 ${ }^{\circ} \mathrm{C}$; specific conductance: 66.1-470.7 $\left.\mu \mathrm{S} / \mathrm{cm}\right)$, circumneutral $\mathrm{pH}(\mathrm{pH}: 6.94-8.16)$, and oxygen undersaturation (dissolved oxygen: 2.9-8.7 mg/L). Detailed physicochemical data and representative photos of each spring are included in Supplementary Tables 1-4 and Supplementary Figure 7.

An HCA incorporating major ions, $\mathrm{Si}$, and specific conductance data divided spring waters into the two major geochemical groups (Figure 1B). Student's t-tests were utilized to assess specific geochemical differences between the groups for $\sim 60$ physical and chemical analytes. This revealed that the two spring groups possessed significantly different $\mathrm{Ca}^{2+} / \mathrm{Na}^{+}$molar ratios $\left(\mathrm{p}\right.$-value $\left.=3.1 * 10^{-5}\right), \mathrm{Ca}^{2+} / \mathrm{Mg}^{2+}$ molar ratios ( $p$-value $=0.05)$, divalent to monovalent cation ion ratios $(\mathrm{D} / \mathrm{M} ; \mathrm{p}$-value $=0.001)$, and slopes of the spring emergences $(p$-value $=0.004)$ (Supplementary Table 1$)$. Spring geochemistry in both groups reflected recharge through granitoid rock, which predominates the high-elevation recharge zones in the Sierra Nevada. All spring waters also contained an excess of calcium $\left(\mathrm{Ca}^{2+} / \mathrm{Na}^{+}>0.6\right)$ over that expected for plagioclase weathering alone $\left(\mathrm{Ca}^{2+} / \mathrm{Na}^{+}\right.$of $0.4-0.6$; Figure $\left.1 \mathrm{C}\right)$, consistent with dissolution of

disseminated calcite during recharge $\mathrm{e}^{[41,74,75,76,77]}$. However, only one spring group contained an "excess of excess calcium", with significantly higher $\mathrm{Ca}^{2+} / \mathrm{Na}^{+}$ratios $\left(\mathrm{Ca}^{2+} / \mathrm{Na}^{+}>1.5\right)$. This "excess of excess calcium" signature has recently been interpreted to reflect roof pendant weathering ${ }^{[41]}$, which is consistent with the location of the five springs with these signatures. Two emerge downgradient from the Pine Creek Pendant units near Mt. Tom (Elderberry Canyon spring, IES-033) and Wheeler Crest (Wells Meadow B spring, IES-038), whereas the three remaining springs emerge downgradient of unnamed roof pendants near Lookout Point (Supplementary Figure 8). For simplicity, the spring group with "excess of excess calcium" signatures is hereafter referred to as "roof pendant springs", whereas those with lower $\mathrm{Ca}^{2+} / \mathrm{Na}^{+}$ ratios are hereafter referred to as "granitic springs".

\section{Roof Pendant Springs Host More Diverse Benthic Macroinvertebrate Communities}

Following quality filtering, 843,111 total $16 S$ rRNA gene sequences from bacteria and archaea were recovered from 39 benthic samples, with an average of 21,618 reads per sample. A total of 14,523 unique ASVs were recovered, approximating the species richness of prokaryotes in these samples, and accounting for 52 bacterial phyla and nine archaeal phyla. Rarefaction curves for all benthic microbial 
community samples reached an asymptote between 10,000-15,000 sequences, indicating adequate sequencing depth for robust comparisons among springs and spring groups (Supplementary Figure 9). A total of 2,660 individual BMls were identified across ten composite benthic samples with an average of 266 BMIs per sample. From the BMI community data, four phyla, seven classes, 16 orders, 47 families, and 85 genera were identified across the ten springs.

Several a-diversity metrics were calculated to determine whether geochemical grouping influenced diversity (Figure 2; Supplementary Tables 5-7). No significant differences in benthic microbial diversity were observed between roof pendant and granitic springs (Figure 2A-D; Supplementary Table 5; Supplementary Table 7). In contrast, BMI a-diversity was higher in roof pendant springs for richness (observed count ANOVA, p-value $=0.009$ ), evenness (Gini-Simpson Index, Kruskal-Wallis Test, p-value $=$ 0.01 ), and diversity (Shannon Index, Kruskal-Wallis Test, p-value $=0.01$ ) (Figure 2E-G; Supplementary Tables $6+7)$.

Subtle Geochemical Differences Alter Community Composition of Benthic Microbes and Macroinvertebrates

NMDS ordinations based on Bray-Curtis dissimilarity for both prokaryotes and BMIs demonstrated that spring groups hosted unique communities (Figure 3). Significant differences were observed in both microbial $(\mathrm{R}$-value $=0.28$, $\mathrm{p}$-value $=0.001)$ and BMI $(\mathrm{R}$-value $=0.65, \mathrm{p}$-value $=0.009)$ communities, with samples clustering on the ordination according to geochemical grouping. Geographic distance was not correlated with Bray-Curtis community dissimilarity of microbial (Mantel test, $p$-value $=0.11$ ) or macroinvertebrate communities (Mantel test, $p$-value $=0.08)($ Supplementary Table S8).

Covariates of community dissimilarity ( $p$-value $\leq 0.05$ ) identified among the $\sim 60$ physical and chemical analytes were fitted to the NMDS ordinations to visualize correlates of community dissimilarity (Figure 3 ). $\mathrm{Ca}^{2+} \mathrm{Na}^{+}, \mathrm{Ca}^{2+} / \mathrm{Mg}^{2+}$, and $\mathrm{D} / \mathrm{M}$ ratios correlated with microbial and $\mathrm{BMI}$ communities from roof pendant springs, reflecting weathering of the roof pendants during recharge. Additional vectors corresponding to spring or sediment characteristics and landscape placement were also significant. In roof pendant springs, current velocity correlated with both communities, whereas slopes of the spring emergences and cobble percentage correlated only with microbial communities and gravel percentage only with BMI communities. In granitic springs, spring water residence time and muck percentage correlated with both communities.

Many analytes correlated only with microbial community dissimilarity (Figure 3A). Temperature and various ions/trace elements (vectors \#12-\#27) correlated with the two oldest springs, specifically, Spring along Hogback Ck. A (IES-024, 1,850 years in age) and Lubken Canyon Spring 2 (IES-054. 1,270 years in age) of the granitic springs group. Mo, $\mathrm{Al}$, and sand percentage correlated with microbial communities in the granitic spring Unnamed Pine Creek Spring (IES-039). Wetted width and V correlated with microbial communities in the granitic spring Unnamed Spring north of Red Mountain (IES-029). 
Benthic microbial communities were compromised predominantly of Proteobacteria ( $46.1 \%$ of the microbial community on average), Bacteroidetes (10.5\%), Acidobacteria (6.9\%), Actinobacteria (5.1\%), Verrucomicrobia (5.1\%), Chloroflexi (4.1\%), Planctomycetes (3.8\%), Nitrospirae (3.7\%), and Cyanobacteria (3.6\%) and other phyla at varying relative abundances across individual springs (Supplementary Figure 10). Relative abundance bar plots representing benthic microbial communities at various taxonomic levels are included in the supplement (Supplementary Figures 11-13).

LEfSe analysis of benthic microbial communities revealed 408 taxa (8 phyla, 33 classes, 69 orders, 111 families, and 187 genera) that were significantly ( $p$-value $\leq 0.05$ ) enriched in either roof pendant springs or granitic springs (Figure 4). Several phyla and one class with multiple child taxa were enriched in roof pendant springs: phyla Acidobacteria (four classes, three orders), Planctomycetes (three classes, four orders), Gemmatimonadetes (three classes, three orders), and Actinobacteria (one class, four orders), and the class Alphaproteobacteria (six orders) (Figure 4A). In granitic springs, only the archaeal phylum Euryarchaeota (two classes, three orders) had multiple classes or orders enriched. Granitic springs were also enriched with several bacterial classes with fewer child taxa, namely Clostridia (one order), Endomicrobia (one order), and Gracilibacteria (one order), and bacterial orders Bacteroidales, Beggiatoales, Chromatiales, Desulfobacterales, Desulfuromonadales, and Methylococcales.

The enriched families and genera suggest a unique set of physiological traits associated with each geochemical group (Figure 4B-C). Roof pendant springs were enriched with aerobic/facultatively anaerobic genera predominantly from Alphaproteobacteria (eight families, 11 genera), Actinobacteria (eight families, eight genera), Planctomycetes (three families, two genera), and Acidobacteria (two families, two genera). These taxa included those known to produce prosthecae/stalks and holdfasts in the Alphaproteobacteria and Planctomycetes, such as Bauldia, Hirschia, Gemmata, Pedomicrobium, Pir4 lineage (family: Pirellulaceae), Schlesneriaceae, Planctomyces, Pedomicrobium, Hyphomicrobiaceae, Micropepsaceae, SWB02 (family: Hyphomonadaceae), and Rhodomicrobium. Granitic springs were instead enriched with anaerobes, including fermenters in the Clostridia (one family, four genera), sulfate reducers in the Deltaproteobacteria (five families, eight genera), and methanogenic archaea within the Euryarchaeota (three families, three genera). Aerobic bacteria included methylotrophic bacteria in the Gammaproteobacteria (six families, four genera) and sulfide oxidizers in the Beggiatoaceae (Figure 4C). A full list of significant results from the LEfSe analysis can be found in the supplement (Supplementary Table 9).

BMI communities differ in composition at multiple taxonomic levels (Supplementary Figure 14; Figure 5). At the genus level, communities in roof pendant springs were relatively even and dominated by vagile insect taxa, such as Lepidostoma, Optioservus, Gumaga, Malenka, Ironodes, Baetis, Argia, and Enchytraeidae with additional populations of lower abundance taxa. These taxa are primarily shredders, collector-gatherers, and predators. Pyrgulopsis was present in only one roof pendant spring, Elderberry Canyon Spring (IES-033), where it was the most abundant taxon. In contrast, granitic spring BMI communities were dominated by Pyrgulopsis and stress-tolerant, non-insect taxa, mainly Pisidium and Hyalella, with less abundant populations of the insect taxa Argia, Optioservus, and Tricladida. 
A co-occurrence network revealed 112 significant ( $p$-value $<0.05$ ) positive correlations between microbes and BMIs (Figure 6). Cluster 1 is a large network highlighting positive correlations between microbial and $\mathrm{BMI}$ genera in roof pendant springs. BMI genera belonging to shredder, collector-gatherer, or predator feeding groups comprised central nodes with high connectivity to microbial taxa, inferring key roles for these taxa in trophic interactions (Figure 6A): Ironodes (14 network connections), Gumaga (13), Baetis (11), Malenka (7), and Pentaneura (7). Prosthecate/stalked bacteria in Cluster 1 included Hyphomicrobium, Pedomicrobium, Pirellula/Pirellulaceae, Gemmataceae, and SWB02 (Hyphomonadaceae). Almost all bacteria in this cluster were aerobic or facultatively anaerobic, while most BMIs were vagile insects with low to moderate stress tolerance values (Figure 6B).

Much less connectivity between microbes and BMls was observed in granitic spring communities (Clusters 2-4), inferring looser trophic associations (Figure 6). In Clusters 2-4 no stalked/holdfast bacteria were observed, unlike Cluster 1 (Figure 6A). BMI genera in Cluster 2 included three functional feeding groups, collector-gatherers (Hyalella), collector-filterers (Pisidium), and predators (Tricladida); both Cluster 3 and Cluster 4 were represented by the scrapers Pyrgulopsis and Zaitzevia. No shredders were present in the granitic spring clusters. The largest network was Cluster 2, with correlations between the stresstolerant BMI genera Pisidium, Hyalella, and Tricladida and a mixture of aerobic/facultatively anaerobic (Dechloromonas and Lacunisphaera), methylotrophic (Crenothrix and Methyloglobulus), and anaerobic (Anaeromyxobacter and Geobacter) bacteria (Figure 6B). In Cluster 3, Zaitzevia was positively associated with the aerobic, ammonia-oxidizing archaeon Candidatus Nitrosoarchaeum and an unclassified genus in the Anaerolineaceae. Cluster 4 included Pyrgulopsis and the cyanobacterium Pleurocapsa PCC-7319 and an unclassified genus of Chitinophagaceae.

\section{Discussion}

We documented distinct communities of benthic microorganisms and macroinvertebrates in springs that recharge through Paleozoic roof pendants thousands of meters upslope from the spring emergences. Weathering of these roof pendants imparts an "excess of excess calcium" and an increased proportion of dissolved calcium compared to other ions ${ }^{[41]}$ that may promote initial microbial attachment to the negatively charged granitic substrate and enhance biofilm maturation within the spring benthos. Biofilms are multispecies surface-attached microbial communities embedded in a three-dimensional matrix of endogenously produced EPS composed of polysaccharides, proteins, extracellular DNA, and lipids ${ }^{[78]}$. Biofilm formation begins with the development of a surface coating comprised of biological material from the surrounding environment, known as a conditioning film, that enhances the initial adhesion of microbial cells ${ }^{[79]}$. Divalent cations are known to reduce electrostatic repulsion by forming cross-bridges between negatively charged surfaces and negatively charged cell surface structures, such as phospholipids, glycolipids, and teichoic acids ${ }^{[80,81]}$. The presence of divalent cations can also promote the expression and functionality of specific adhesins in a variety of bacterial species ${ }^{[80,81]}$. During biofilm 
maturation, the formation of non-specific cation cross-bridges between cells and EPS enhances the thickness and structural stability of the biofilm and traps nutrients ${ }^{[81,82]}$. Most research on the role of $\mathrm{Ca}^{2+}$ or other divalent cations in microbial attachment and biofilm formation focuses on ionic strength, yet neither $\mathrm{Ca}^{2+}$ concentration nor total ionic strength accounted for the community structures observed in the roof pendant springs. Instead, these patterns were attributed to differences in valence, specifically $\mathrm{Ca}^{2+} / \mathrm{Na}^{+}$ratios.

A past study showed that $\mathrm{Ca}^{2+} / \mathrm{Na}^{+}$ratios $>1.0$ resulted in larger microbial flocs (aggregations) in activated sludge compared to those with ratios $<1.0^{[83]}$. Although our study focuses on benthic biofilms rather than suspended aggregates, we also identified $\mathrm{Ca}^{2+} / \mathrm{Na}^{+}$ratios as the strongest correlate of community structure and propose that high $\mathrm{Ca}^{2+} / \mathrm{Na}^{+}$ratios promote microbial attachment to granite in these fast-flowing springs. Only springs in the roof pendant group possessed $\mathrm{Ca}^{2+} / \mathrm{Na}^{+}$ratios above 1.0 (average: $1.5 \pm 0.12$ ), and these springs were enriched with unrelated lineages of bacteria often associated with biofilms, including members of the phyla Acidobacteria, Planctomycetes,

Gemmatimonadetes, and Actinobacteria, and the class Alphaproteobacteria ${ }^{[84]}$ (Figure 4). Several of the enriched Alphaproteobacteria and Planctomycetes also produce prosthecae and/or stalks, many of these structures are tipped with holdfast adhesins, which are secreted polysaccharides used for attachment and biofilm formation ${ }^{[85,86,87]}$. Previous work with a purified polysaccharide holdfast adhesin (fr2ps) from Hyphomonas MHS-3 demonstrated a critical role for $\mathrm{Ca}^{2+}$ cross-bridging in the initial binding of this adhesin to germanium oxide surfaces ${ }^{[8]}$. We postulate that the higher abundance of biofilm-associated and prosthecate/stalked bacteria in roof pendant springs is a response to the molar excess of $\mathrm{Ca}^{2+}$, which may promote microbial surface attachment and biofilm development. In flowing aquatic systems such as spring runs or streams, surface attachment and biofilm formation are necessary for benthic microorganisms as it prevents cells from being flushed out of the system. As cells attach and settle in the system, endogenous nutrient cycling is increased, promoting the development of metabolically diverse assemblages and enhances trophic interactions ${ }^{[84,89]}$.

Calcium may also be important for structuring BMI communities, as observed in other aquatic systems ${ }^{[90,}$ 91], although most studies do not address mechanisms for this relationship. We propose that $\mathrm{Ca}^{2+}$ may structure these BMI communities indirectly through trophic interactions with microbial biofilms (as discussed below) or directly by influencing BMI physiology. For example, $\mathrm{Ca}^{2+}$ is present at a 1:1 molar ratio with phosphoserines in the major silk adhesin of caddisfly larvae, where it forms stabilizing cation cross-bridges between antiparallel $\beta$-sheets ${ }^{\left[{ }^{[2]}\right]}$. This adhesin is initially secreted from the silk gland as a fluid that is then solidified and stabilized into metallofibers by $\mathrm{Ca}^{2+}$ absorbed from the surrounding environment ${ }^{[92]}$. When $\mathrm{Ca}^{2+}$ is replaced with $\mathrm{Na}^{+}$, the $\beta$-sheets in the stabilized silk adhesin revert to random coils, destroying the structure and adhesive properties of the silk ${ }^{[93]}$. $\mathrm{Ca}^{2+}$-stabilized silk adhesins are necessary for substrate attachment and construction of composite cases comprised of stones and detritus that are utilized by the larvae for protection and in food gathering ${ }^{[94]}$. In our study, the caddisflies Gumaga and Lepidostoma were dominant BMI taxa in roof pendant springs but were nearly absent from 
granitic springs, which may be a direct response to $\mathrm{Ca}^{2+} / \mathrm{Na}^{+}$ratios. Given their roles as shredders, Gumaga, Lepidostoma, and other enriched taxa, such as the stonefly Malenka, likely interact extensively for the synergistic degradation of allochthonous organic matter, explaining the well-connected microbeBMI network in roof pendant springs (Figure 6).

Allochthonous organic matter is the dominant source of organic carbon in most springs and streams ${ }^{[95,}$ $96,97]$ and is typically derived from the debris of riparian vegetation, collectively referred to as CPOM (>1 $\mathrm{mm}$ in particle size ${ }^{[32]}$. BMI shredders and microorganisms are responsible for the synergistic breakdown of CPOM into FPOM ( $<1 \mathrm{~mm}$ in particle size) that is then ingested by BMI collector-gatherers ${ }^{[32,97]}$. Both shredders and collector-gatherers preferentially feed on organic matter that has been colonized by microbial biofilms ${ }^{[32,98]}$ due to the stoichiometrically favorable nutrient ratios (C:N and C:P) of microorganisms over plant biomass ${ }^{[32,97]}$. EPS, which often comprise more biomass than cells in biofilms, are an additional food source for BMIs, further facilitating the transfer of energy to higher trophic levels ${ }^{[99,100]}$. Thus, we propose that $\mathrm{Ca}^{2+}$-enriched populations of biofilm-associated and stalked/holdfast bacteria in roof pendant springs exert a bottom-up control on shredders and collectorgatherers as a food source and metabolic partner. Subsequently, shredders may enforce top-down controls on CPOM-associated biofilms by increasing CPOM surface area and the availability of microbial attachment sites ${ }^{[32,97,101]}$ or potentially through selective grazing of larger microbial cells/filaments in the biofilm.

An alternative top-down control on benthic microbial biofilms could potentially be driven by the hydrobiid gastropod genus Pyrgulopsis, given their high population density in all granitic springs and their feeding behavior as scrapers. Our results, however, do not support this hypothesis. Only the roof pendant Elderberry Canyon Spring (IES-033) hosted Pyrgulopsis (Figure 5). Despite the presence of Pyrgulopsis, the microbial and BMI communities in the Elderberry Canyon Spring clustered with the other roof pendant spring communities on the NMDS plots (Figure 3). Furthermore, the microbial communities in springs with or without Pyrgulopsis were not significantly different (ANOSIM: R $=0.06, \mathrm{p}$-value $=0.12$ ). Additionally, Elderberry Canyon Spring hosts enriched populations of biofilm-associated and stalked/holdfast bacteria, like other roof pendant springs. Altogether, we did not identify any evidence consistent with top-down control of biofilm-associated and stalked/holdfast bacteria through grazing by Pyrgulopsis.

In aquatic environments, current velocity is one of the most important factors determining the grain size of benthic substrate ${ }^{[102]}$, along with microbial| ${ }^{[103]}$ and $\mathrm{BMI}^{[26,27]}$ communities. Fine grain sizes that dominate in low-flow springs slows oxygen diffusion, selecting for anaerobic microorganisms and spatially controlled redox cycles of $\mathrm{C}, \mathrm{N}, \mathrm{S}$, and other elements ${ }^{[104]}$. The roof pendant-recharged springs tended to have higher currently velocities and were appropriately enriched with aerobic and facultatively anaerobic bacteria. This spring group also contained diverse, low-moderate stress tolerant insect taxa, in line with the coarser substrates found in these springs. In contrast, the generally slower flowing graniterecharged springs were enriched with anaerobes such as obligate fermenters, sulfate reducers, and 
methanogens, as well as the corresponding chemolithotrophs-sulfur oxidizers and methylotrophs (Figure 4). The granite-recharged springs also hosted highly uneven BMI communities dominated by moderate-high stress tolerant non-insect taxa. Despite these trends suggesting the importance of current velocity, there were a couple exceptions. The current velocity at South Harry Birch Spring (IES-043) was among the lowest of any of the springs, whereas Unnamed Spring north of Red Mountain (IES-029) was among the fastest. Even with their exceptional current velocities, both microbial and BMI communities in these springs clustered with their respective geochemical group (Figure 3), underscoring the hypothesis that the roof pendant-driven molar excesses of $\mathrm{Ca}^{2+}$ is the primary factor structuring these communities.

A third alternative environmental control was spring water residence time, or the time elapsed between recharge and discharge. In general, older residence times are associated with warmer, more geochemically evolved water ${ }^{[105,106]}$. The specific physicochemical effects associated with increased residence time are highlighted as important factors structuring microbial communities in groundwater-fed ecosystems, such as streams ${ }^{[16]}$. and aquifers ${ }^{[107,108]}$. Additionally, temperature and salinity are wellrecognized drivers of microbial community ecology across multiple biomes ${ }^{[18,19,20]}$. In our study, spring residence time estimates varied from 40-200 years for most springs to over 1,200 years for Lubken Canyon Spring 2 (IES-054) and Spring along Hogback Ck. A (IES-024). Residence time correlated with both communities and trended with vectors for specific conductivity and many individual ions, diagnostic of increased water-rock interactions (Figure 3). These relationships, however, were subordinate to the geochemical groupings. Ultimately, although residence time appears to play some role in structuring the ecology of these communities, the molar excess of $\mathrm{Ca}^{2+}$ imparted by the roof pendant remains the dominant factor.

\section{Conclusions}

This study answers the call for implementation of an integrated ecohydrogeology approach to better understand spring ecosystems ${ }^{[2,3,4]}$. By using this approach, we demonstrate, for the first time, a strong ecological response of desert spring benthic communities across multiple trophic levels to subtle changes in spring geochemistry imparted by recharge through roof pendants. This response is striking given the overall geochemical similarity of these springs, as the recharge path for both groups is predominantly through granitoid rock. The tightly connected community responses to roof pendantderived $\mathrm{Ca}^{2+} / \mathrm{Na}^{+}$ratios-namely enhanced benthic biofilms and abundant, diverse populations of shredder and collector-gatherer insect taxa in roof pendant springs-likely increase the flux of biomass and energy through the benthos and beyond to higher trophic levels in these springs as well as associated riparian areas. These and other ecological responses to subtle differences in spring geochemistry should be further explored in the Sierra Nevada and beyond, preferably within an ecohydrogeological framework, to refine our understanding of the connections between spring hydrogeochemistry and ecology.

\section{Declarations}




\section{Data availability}

Unprocessed reads are available in the NCBI Sequence Read Archive (SRA) under the accession PRJNA788156. Scripts for relevant analyses can be found in the github repository hedlundb/SNRoofPendant.

\section{Acknowledgements}

This research was supported through the National Science Foundation (EAR 1516680, EAR 1516127, EAR 1516698, EAR 1516488, and EAR 1516679). We thank all members of this interdisciplinary project for intellectual support, Jef Jaeger for careful editing, and Lynn Owens for assistance preparing DNA extracts for Illumina 16S rRNA gene sequencing. Additionally, we thank Los Angeles Department of Water and Power (James Yannotta and Jeff Nordin) and Inyo National Forest (Rosalie Herrera and Ed Armenta; Permit \#WMD16014) for their permission to visit and sample these incredible ecosystems.

\section{Author information}

\section{Contributions}

This study was conceived by ADF, KP, ZM, BPH, and DS. Samples for geochemistry measurements were collected and processed by ADF, BPH, ZM, LR, and MF. Microbial samples were collected and processed by $A D F, N T$, and $B P H$, whereas KP and DS collected and processed BMI samples. ADF and BPH worked together to design the figures, but ADF was responsible for generating and editing all figures for the manuscript with assistance from COS. ADF created all tables, which were edited by $\mathrm{BPH}$. All statistical analyses were run by ADF with assistance from COS. ADF wrote the first draft of this manuscript, which was initially edited by BPH. Subsequent drafts were edited by all authors.

\section{Competing interests}

The authors of this manuscript declare that they have no commercial or financial relationships that could serve as a potential conflict of interest to this work.

\section{References}

1. Meinzer, O.E. Outline of ground-water hydrology, with definitions. USGPO U.S. Geol. Surv. WaterSupply Pap. 494. https://doi.org/10.3133/wsp494 (1923).

2. Cantonati, M. et al. Geological and hydrochemical prerequisites of unexpectedly high biodiversity in spring ecosystems at the landscape level. Sci. Total Environ. 740<bvertical-align:super; $>,</$ bverticalalign:super;> e140157. https://doi.org/10.1016/j.scitotenv.2020.140157 (2020b).

3. Stevens, L.E., Schenk, E.R. and Springer, A.E. Springs ecosystem classification. Ecol. Appl. 31<bvertical-align:super;>,</bvertical-align:super;> e2218. https://doi.org/10.1002/eap.2218 (2021). 
4. Cantonati, M. et al. Ecohydrogeology: The interdisciplinary convergence needed to improve the study and stewardship of springs and other groundwater-dependent habitats, biota, and ecosystems. Ecol. Indic. 110<bvertical-align:super;>,</bvertical-align:super;> e105803. https://doi.org/10.1016/j.ecolind.2019.105803 (2020a).

5. Davis, J.A., Kerezsy, A. and Nicol, S. Springs: conserving perennial water is critical in arid landscapes. Biol. Conserv. 211<bvertical-align:super;>,</bvertical-align:super;> 30-35. https://doi.org/10.1016/j.biocon.2016.12.036 (2017).

6. Shepard, W.D. Desert springs-both rare and endangered. Aquat. Conserv. $3<$ bvertical-align:super;>, </bvertical-align:super;> 351-359. https://doi.org/10.1002/aqc.3270030409 (1993).

7. Myers, M.J. and Resh, V.H. Trichoptera and other macroinvertebrates in springs of the Great Basin: species composition, richness, and distribution. West. N. Am. Nat. 62<bvertical-align:super;>, </bvertical-align:super;> 1-13. https://www.jstor.org/stable/41717152 (2002).

8. Meixner, T. et al. Implications of projected climate change for groundwater recharge in the western United States. J. Hydrol. 534<bvertical-align:super;>,</bvertical-align:super;> 124-138. https://doi.org/10.1016/j.jhydrol.2015.12.027 (2016).

9. Bodelier, P.L.E. Toward understanding, managing, and protecting microbial ecosystems. Front. Microbiol. 2<bvertical-align:super;>,</bvertical-align:super;> 80. https://doi.org/10.3389/fmicb.2011.00080 (2011).

10. Lennon, J.T. and Locey, K.J. More support for Earth's massive microbiome. Biol. Direct $15<$ bverticalalign:super;>></bvertical-align:super;><bvertical-align:super;></bvertical-align:super;>1-6. https://doi.org/10.1186/s13062-020-00261-8 (2020).

11. Souza, V. et al. An endangered oasis of aquatic microbial biodiversity in the Chihuahuan desert. Proc. Natl. Acad. Sci. <bvertical-align:super;>103</bvertical-align:super;><bvertical-align:super;>,</bverticalalign:super;><bvertical-align:super;></bvertical-align:super;>6565-6570. https://doi.org/10.1073/pnas.0601434103 (2006).

12. Breitbart, M. et al. Metagenomic and stable isotopic analyses of modern freshwater microbialites in Cuatro Ciénegas, Mexico. Environ. Microbiol. 11<bvertical-align:super;>,</bvertical-align:super;> <bvertical-align:super;></bvertical-align:super;>16-34. https://doi.org/10.1111/j.14622920.2008.01725.x (2009).

13. Souza, V. et al. The lost world of Cuatro Cienegas Basin, a relictual bacterial niche in a desert oasis. eLife 7<bvertical-align:super;>></bvertical-align:super;> e38278. https://doi.org/10.7554/eLife.38278 (2018).

14. Elser, J.J. et al. Effects of phosphorus enrichment and grazing snails on modern stromatolitic microbial communities. Freshw. Biol. 50<bvertical-align:super;></bvertical-align:super;> 1808-1825. https://doi.org/10.1111/j.1365-2427.2005.01451.x (2005).

15. Kaestli, M., Munksgaard, N., Gibb, K. and Davis, J. Microbial diversity and distribution differ between water column and biofilm assemblages in arid-land waterbodies. Freshw. Sci. $\mathbf{3 8}<$ bverticalalign:super;>,</bvertical-align:super;> 869-882. https://doi.org/10.1086/706106 (2019). 
16. Zeglin, L.H., Crenshaw, C.L., Dahm, C.N., Sheibley, R.W. and Takacs-Vesbach, C.D. Watershed hydrology and salinity, but not nutrient chemistry, are associated with arid-land stream microbial diversity. Freshw. Sci. 38<bvertical-align:super;>,</bvertical-align:super;> 77-91. https://doi.org/10.1086/701597 (2019).

17. Fierer, N. and Jackson, R.B. The diversity and biogeography of soil bacterial communities. Proc. Natl. Acad. Sci. <bvertical-align:super;>103</bvertical-align:super;><bvertical-align:super;>,</bverticalalign:super;><bvertical-align:super;></bvertical-align:super;>626-631. https://doi.org/10.1073/pnas.0507535103 (2006).

18. Lozupone, C.A. and Knight, R. Global patterns in bacterial diversity. Proc. Natl. Acad. Sci. <bverticalalign:super;>104</bvertical-align:super;><bvertical-align:super;>,</bvertical-align:super;> 1143611440. https://doi.org/10.1073/pnas.0611525104 (2007).

19. Thompson, L.R. et al. A communal catalogue reveals Earth's multiscale microbial diversity. Nature 55<bvertical-align:super;>,</bvertical-align:super;><bvertical-align:super;></bverticalalign:super;>457-463. https://doi.org/10.1038/nature24621 (2017).

20. Power, J.F. et al. Microbial biogeography of 925 geothermal springs in New Zealand. Nat. Comm. 9<bvertical-align:super;>,</bvertical-align:super;> e2876. https://doi.org/10.1038/s41467-018-05020y (2018).

21. Barquín, J. and Death, R.G. Spatial patterns of macroinvertebrate diversity in New Zealand springbrooks and rhithral streams. J. N. Am. Benthol. Soc. 25<bvertical-align:super;>,</bverticalalign:super;> 768-786. https://doi.org/10.1899/0887-3593(2006)025[0768:SPOMDI]2.0.C0;2 (2006).

22. Seidel, R.A., Lang, B.K. and Berg, D.J. Salinity tolerance as a potential driver of ecological speciation in amphipods (Gammarus spp.) from the northern Chihuahuan Desert. J. N. Am. Benthol. Soc. 29<bvertical-align:super;>,</bvertical-align:super;><bvertical-align:super;></bverticalalign:super;>1161-1169. https://doi.org/10.1899/09-098.1 (2010).

23. Glazier, D.S. Temperature affects food-chain length and macroinvertebrate species richness in spring ecosystems. Freshw. Sci. 31<bvertical-align:super;>,</bvertical-align:super;> 575-585. https://doi.org/10.1899/11.058.1 (2012).

24. Quinn, J.M. and Hickey, C.W. Magnitude of effects of substrate particle size, recent flooding, and catchment development on benthic invertebrates in 88 New Zealand rivers. N. Z. J. Mar. 24<bverticalalign:super;>,</bvertical-align:super;><bvertical-align:super;></bvertical-align:super;>411-427. https://doi.org/10.1080/00288330.1990.9516433 (1990).

25. Duan, X.H., Wang, Z.Y., Xu, M.Z., and Zhang, K. Effect of streambed sediment on benthic ecology. Int. J. Sediment Res. 24<bvertical-align:super;>,</bvertical-align:super;> 325-338. https://doi.org/10.1016/S1001-6279(10)60007-8 (2009).

26. Rempel, L.L., Richardson, J.S. and Healey, M.C. Macroinvertebrate community structure along gradients of hydraulic and sedimentary conditions in a large gravel-bed river. Freshw. Biol. 
45<bvertical-align:super;>,</bvertical-align:super;> 57-73. https://doi.org/10.1046/j.13652427.2000.00617.x (2000).

27. Mérigoux, S. and Dolédec, S. Hydraulic requirements of stream communities: a case study on invertebrates. Freshw. Biol. 49<bvertical-align:super;>,</bvertical-align:super;><bvertical-align:super;> </bvertical-align:super;>600-613. https://doi.org/10.1111/j.1365-2427.2004.01214.x (2004).

28. Covich, A.P., Palmer, M.A., and Crowl, T.A. The role of benthic invertebrate species in freshwater ecosystems: zoobenthic species influence energy flows and nutrient cycling. BioScience $49<$ bverticalalign:super;>></bvertical-align:super;><bvertical-align:super;></bvertical-align:super;>119-127. https://doi.org/10.2307/1313537 (1999).

29. Taylor, J. M., Vanni, M. J., and Flecker, A. S. Top-down and bottom-up interactions in freshwater ecosystems: emerging complexities. In: Trophic ecology: bottom-up and top-down interactions across aquatic and terrestrial systems (eds Hanley, T. C. and La Pierre, K. J.) 55-85. (Cambridge University Press, 2015).

30. Krumins, J.A. et al. Soil and freshwater and marine sediment food webs: their structure and function. Bioscience 63<bvertical-align:super;>,</bvertical-align:super;> 35-42. https://doi.org/10.1525/bio.2013.63.1.8 (2013).

31. Battin, T.J., Besemer, K., Bengtsson, M.M., Romani, A.M., and Packmann, A.I. The ecology and biogeochemistry of stream biofilms. Nat. Rev. Microbiol. 14<bvertical-align:super;>,</bverticalalign:super;> 251-263. https://doi.org/10.1038/nrmicro.2016.15 (2016).

32. Cummins, K.W. Structure and function of stream ecosystems. BioScience $\mathbf{2 4 < b v e r t i c a l - a l i g n : s u p e r ; > ,}$ </bvertical-align:super;> 631-641. https://doi.org/10.2307/1296676 (1974).

33. Cummins, K.W. and Klug, M.J. Feeding ecology of stream invertebrates. Annu. Rev. Ecol. Evol. Syst. 10<bvertical-align:super;>,</bvertical-align:super;> 147-172. https://doi.org/10.1146/annurev.es.10.110179.001051 (1979).

34. Hollett, K.J., Danskin, W.R., McCaffrey, W.F. and Walti, C.L. Geology and water resources of Owens Valley, California. USGPO U.S. Geol. Surv. Water-Supply Pap. 2370-B. https://doi.org/10.3133/wsp2370B (1991).

35. Moore, J.G. Geology of the mount Pinchot quadrangle, southern Sierra Nevada, California U.S. Geol. Surv. Bull. 1138. https://doi.org/10.3133/b1130 (1963).

36. Bateman, P.C., Pakiser, L.C. and Kane, M.F. Geology and tungsten mineralization of the Bishop district, California, with a section on gravity study of Owens Valley and a section on seismic profile. U.S. Geol. Surv. Professional Paper 470. https://doi.org/10.3133/pp470 (1965)

37. Stevens, C.H. and Greene, D.C. Stratigraphy, depositional history, and tectonic evolution of Paleozoic continental-margin rocks in roof pendants of the eastern Sierra Nevada, California. GSA Bulletin 111<bvertical-align:super;>,</bvertical-align:super;> 919-933. https://doi.org/10.1130/00167606(1999)111<0919:SDHATE>2.3.C0;2 (1999).

38. Duell Jr., L.F.W. Estimates of evapotranspiration in alkaline scrub and meadow communities of Owens Valley, California, using the Bowen-ratio, eddy-correlation, and Penman-combination methods. 
USGPO U.S. Geol. Surv. Water-Supply Pap. 2370-E. https://doi.org/10.3133/wsp2370E (1990).

39. Miller-Coleman, R.L. et al. Korarchaeota diversity, biogeography, and abundance in Yellowstone and Great Basin hot springs and ecological niche modeling based on machine learning. PlOS ONE 7<bvertical-align:super;>,</bvertical-align:super;> e35964.

https://doi.org/10.1371/journal.pone.0035964 (2012).

40. Li, G., Ding, H. and Ji, J. SuperPCA: supervised principal component analysis. R package version 0.3.0. https://CRAN.R-project.org/package=SuperPCA (2020).

41. Meyers, Z., Rademacher, L.K., Frisbee, M.D., Warix, S.R. Extending classical geochemical weathering studies through the mountain block: The effect of increasing scale on geochemical evolution in the Sierra Nevada (CA). (2022). Submitted.

42. Gleason, C.L. et al. Hydrogeology of desert springs in the Panamint Range, California, USA: Geologic controls on the geochemical kinetics, flowpaths, and mean residence times of springs. Hydrol. Process 34<bvertical-align:super;>,</bvertical-align:super;> 2923-2948.

https://doi.org/10.1002/hyp.13776 (2020).

43. Jurgens, B.C., Böhlke, J.K., Eberts, S.M. TracerLPM (Version 1): an Excel® workbook for interpreting groundwater age distributions from environmental tracer data. U.S. Geol. Surv. Techn. Meth. Report 4F3. https://doi.org/10.3133/tm4F3 (2012).

44. Han, L.F., Plummer, L.N. and Aggarwal, P. A graphical method to evaluate predominant geochemical processes occurring in groundwater systems for radiocarbon dating. Chem. Geol. 318<bverticalalign:super;>,</bvertical-align:super;> 88-112. https://doi.org/10.1016/j.chemgeo.2012.05.004 (2012).

45. Parkhurst, D.L., Charlton, S.R. NetpathXL - An Excel® interface to the program NETPATH. U.S. Geol. Surv. Tech. and Meth. Report 6-A26. (2008).

46. Vogel, J. Groningen radiocarbon dates IX. Radiocarbon 12<bvertical-align:super;>,</bverticalalign:super;> 444-471. https://doi.org/10.1017/S0033822200008183 (1970).

47. Pearson, F.J., Hanshaw, B.B. Sources of dissolved carbonate species in groundwater and their effects on carbon-14 dating. In: Isotope Hydrology IAEA, 271-286. (1970).

48. Fontes, J.C. and Garnier, J.M. Determination of the initial $14 \mathrm{C}$ activity of the total dissolved carbon: A review of the existing models and a new approach. Water Resour. Res. 15<bvertical-align:super;>, </bvertical-align:super;> 399-413. https://doi.org/10.1029/WR015i002p00399 (1979).

49. Han, L.F. and Plummer, L.N. Revision of Fontes \& Garnier's model for the initial $14 \mathrm{C}$ content of dissolved inorganic carbon used in groundwater dating. Chem. Geol. 351<bvertical-align:super;>, </bvertical-align:super;> 105-114. https://doi.org/10.1016/j.chemgeo.2013.05.011 (2013).

50. Han, L.F. and Plummer, L.N. A review of single-sample-based models and other approaches for radiocarbon dating of dissolved inorganic carbon in groundwater. Earth Sci. Rev. 152<bverticalalign:super;>,</bvertical-align:super;> 119-142. https://doi.org/10.1016/j.earscirev.2015.11.004 (2016). 
51. Apprill, A., McNally, S., Parsons, R., and Weber, L. Minor revision to V4 region SSU rRNA 806R gene primer greatly increases detection of SAR11 bacterioplankton. Aquat. Microb. Ecol. 75<bverticalalign:super;>,</bvertical-align:super;> 129-137. https://doi.org/10.3354/ame01753 (2015).

52. Parada, A.E., Needham, D.M., and Fuhrman, J.A. Every base matters: assessing small subunit rRNA primers for marine microbiomes with mock communities, time series and global field samples.

Environ. Microbiol. 18<bvertical-align:super;>,</bvertical-align:super;><bvertical-align:super;> </bvertical-align:super;>1403-1414. https://doi.org/10.1111/1462-2920.13023 (2016).

53. Bolyen, E. et al. Reproducible, interactive, scalable and extensible microbiome data science using QIIME 2. Nat. Biotechnol. 37<bvertical-align:super;>,</bvertical-align:super;> 852-857. https://doi.org/10.1038/s41587-019-0209-9 (2019).

54. Callahan, B.J., McMurdie, P.J., Rosen, M.J., Han, A.W., Johnson, A.J.A., and Holmes, S. P. DADA2: high-resolution sample inference from Illumina amplicon data. Nat. Methods $13<$ bverticalalign:super;>,</bvertical-align:super;> 581-583. https://doi.org/10.1038/nmeth.3869 (2016).

55. Pruesse, E. et al. SILVA: a comprehensive online resource for quality checked and aligned ribosomal RNA sequence data compatible with ARB. Nucleic Acids Res. 35<bvertical-align:super;>,</bverticalalign:super;> 7188-7196. https://doi.org/10.1093/nar/gkm864 (2007).

56. Bokulich, N.A. et al. Optimizing taxonomic classification of marker-gene amplicon sequences with QIIME 2's q2-feature-classifier plugin. Microbiome 6<bvertical-align:super;>,</bvertical-align:super;> 90. https://doi.org/10.1186/s40168-018-0470-z (2018).

57. Price, M.N., Dehal, P.S. and Arkin, A.P. FastTree 2-approximately maximum-likelihood trees for large alignments. PLoS ONE 5, e9490. https://doi.org/10.1371/journal.pone.0009490 (2010).

58. Glazier, D.S. The fauna of North American temperate cold springs: patterns and hypotheses. Freshw. Biol. 26<bvertical-align:super;>,</bvertical-align:super;> 527-542. https://doi.org/10.1111/j.13652427.1991.tb01417.x (1991).

59. McCabe, D.J. Biological communities in springbrooks. In: Studies in Crenobiology: The biology of springs and springbrooks. 221-228. (Backhuys Publishers, 1998).

60. Vinson, M.R. and Hawkins, C.P. Effects of sampling area and subsampling procedure on comparisons of taxa richness among streams. J. N. Am. Benthol. Soc. 15<bvertical-align:super;>, </bvertical-align:super;> 392-399. https://doi.org/10.2307/1467286 (1996).

61. Dixon, P. VEGAN, a package of R functions for community ecology. J. Vegetation Sci. 14<bverticalalign:super;>,</bvertical-align:super;><bvertical-align:super;></bvertical-align:super;>927-930. https://doi.org/10.1111/j.1654-1103.2003.tb02228.x (2003).

62. Kembel, S.W. et al. Picante: R tools for integrating phylogenies and ecology. Bioinformatics 26<bvertical-align:super;>,</bvertical-align:super;> 1463-1464. https://doi.org/10.1093/bioinformatics/btq166 (2010).

63. Wickham, H. ggplot2: Elegant graphics for data analysis. Springer-Verlag New York. ISBN 978-3-31924277-4. https://ggplot2.tidyverse.org (2016). 
64. McMurdie, P.J. and Holmes, S. phyloseq: an R package for reproducible interactive analysis and graphics of microbiome census data. PLoS ONE 8<bvertical-align:super;>,</bvertical-align:super;> e61217. https://doi.org/10.1371/journal.pone.0061217 (2013).

65. Fox, J. and Weisberg, S. An R companion to applied regression, Third Edition. Sage, Thousand Oaks CA. R package version 3.0-10. https://socialsciences.mcmaster.ca/jfox/Books/Companion/ (2019).

66. de Mendiburu, F. agricolae: statistical procedures for agricultural research. R package version 1.3-3. link: https://CRAN.R-project.org/package=agricolae (2020).

67. Padgham, M. and Sumner, M.D. geodist: fast, dependency-free geodesic distance calculations. R package version 0.0.7. https://CRAN.R-project.org/package=geodist (2021).

68. Yang, C. microbiomeMarker: microbiome biomarker analysis. R package version 0.0.1.9000. https://github.com/yiluheihei/microbiomeMarker. https://doi.org/10.5281/zenodo.3749415. (2020).

69. Harrell, F.E. Jr. Hmisc: Harrell miscellaneous. R package version 4.4-2. link: https://CRAN.Rproject.org/package=Hmisc (2020).

70. Butts, C. network: classes for relational data. R package version 1.16.1, https://CRAN.Rproject.org/package=network. (2020).

71. Butts, C. network: a package for managing relational data in R. J. Stat. Softw. $\mathbf{2 4 < b v e r t i c a l -}$ align:super;>,</bvertical-align:super;><bvertical-align:super;></bvertical-align:super;>1-36. https://doi.org/10.18637/jss.v024.i02 (2008).

72. Briatte, F. ggnet: functions to plot networks with ggplot2. R package version 0.1.0. https://github.com/briatte/ggnet (2021).

73. Mandaville, S.M. Benthic macroinvertebrates in freshwaters: Taxa tolerance values, metrics, and protocols Nova Scotia: Soil \& Water Conservation Society of Metro Halifax. http://www.chebucto.ns.ca/science/SWCS/H-1/tolerance.pdf (2002).

74. Garrels, R.M. and Mackenzie, F.T. Origin of the chemical compositions of some springs and lakes. Adv. Chem. Ser. 67<bvertical-align:super;></bvertical-align:super;> 222-242. https://doi.org/10.1021/ba-1967-0067.ch010 (1967).

75. White, A.F. et al. Chemical weathering in a tropical watershed, Luquillo Mountains, Puerto Rico: I. Long-term versus short-term weathering fluxes. Geochim. Cosmochim. Acta $62<$ bverticalalign:super;>></bvertical-align:super;> 209-226. https://doi.org/10.1016/S0016-7037(97)00335-9 (1998).

76. Blum, J.D., Gazis, C.A., Jacobson, A.D., and Page Chamberlain, C. Carbonate versus silicate weathering in the Raikhot watershed within the High Himalayan Crystalline Series. Geology 26<bvertical-align:super;>></bvertical-align:super;> 411-414. https://doi.org/10.1130/00917613(1998)026<0411:CVSWIT>2.3.C0;2 (1998).

77. Pretti, V.A. and Stewart, B.W. Solute sources and chemical weathering in the Owens Lake watershed, eastern California. Water Resour. Res. 38<bvertical-align:super;>,</bvertical-align:super;> 1127. https://doi.org/10.1029/2001WR000370 (2002). 
78. Flemming, H.C. and Wingender, J. The biofilm matrix. Nature Rev. Microbiol. 8<bvertical-align:super;>, </bvertical-align:super;> 623-633. https://doi.org/10.1038/nrmicro2415 (2010).

79. Berne, C., Ellison, C.K., Ducret, A. and Brun, Y.V. Bacterial adhesion at the single-cell level. Nat. Rev. Microbiol. 16<bvertical-align:super;>></bvertical-align:super;> 616-627. https://doi.org/10.1038/s41579-018-0057-5 (2018).

80. Geesey, G.G., Wigglesworth-Cooksey, B. and Cooksey, K.E. Influence of calcium and other cations on surface adhesion of bacteria and diatoms: a review. Biofouling $15<$ bvertical-align:super; $>,</$ bverticalalign:super;> 195-205. https://doi.org/10.1080/08927010009386310 (2000).

81. Wang, T., Flint, S. and Palmer, J. Magnesium and calcium ions: roles in bacterial cell attachment and biofilm structure maturation. Biofouling 35<bvertical-align:super;>,</bvertical-align:super;> 959-974. https://doi.org/10.1080/08927014.2019.1674811 (2019).

82. Sobeck, D.C. and Higgins, M.J. Examination of three theories for mechanisms of cation-induced bioflocculation. Water Res. 36<bvertical-align:super;>,</bvertical-align:super;> 527-538. https://doi.org/10.1016/S0043-1354(01)00254-8 (2002).

83. Cousin, C.P. and Ganczarczyk, J.J. Effect of calcium ion concentration on the structure of activated sludge flocs. Environ. Technol. 20<bvertical-align:super;>,</bvertical-align:super;><bverticalalign:super;></bvertical-align:super;>1129-1138. https://doi.org/10.1080/09593332008616910 (1999).

84. Besemer, K. Biodiversity, community structure and function of biofilms in stream ecosystems. Res. Microbiol. 166<bvertical-align:super;>,</bvertical-align:super;> 774-781. https://doi.org/10.1016/j.resmic.2015.05.006 (2015).

85. Jogler, M. and Jogler, C. Toward the development of genetic tools for Planctomycetes. In Planctomycetes: cell structure, origins and biology (eds Fuerst J.) 141-164. https://doi.org/10.1007/978-1-62703-502-6_6 (Humana Press, 2013).

86. Berne, C., Ducret, A., Hardy, G. G. \& Brun, Y. V. Adhesins involved in attachment to abiotic surfaces by Gram-negative bacteria. Microbiol. Spectr. <bvertical-align:super;>3</bvertical-align:super;><bverticalalign:super;>,</bvertical-align:super;> MB-0018-2015. https://doi.org/10.1128/9781555817466.ch9 (2015).

87. Fritts, R.K., LaSarre, B., Stoner, A.M., Posto, A.L. and McKinlay, J.B. A Rhizobiales-specific unipolar polysaccharide adhesin contributes to Rhodopseudomonas palustris biofilm formation across diverse photoheterotrophic conditions. Appl. Environ. Microbiol. 83<bvertical-align:super;>,</bverticalalign:super;> e03035-16. https://doi.org/10.1128/AEM.03035-16 (2017).

88. Bhosle, N., Suci, P.A., Baty, A.M., Weiner, R.M. and Geesey, G.G. Influence of divalent cations and pH on adsorption of a bacterial polysaccharide adhesin. J. Colloid Interface Sci. $\mathbf{2 0 5 < b v e r t i c a l - ~}$ align:super;>,</bvertical-align:super;> 89-96. https://doi.org/10.1006/jcis.1998.5597 (1998).

89. Battin, T.J. et al. Biophysical controls on organic carbon fluxes in fluvial networks. Nat. Geosci. 1<bvertical-align:super;>,</bvertical-align:super;> 95-100. https://doi.org/10.1038/ngeo101 (2008). 
90. Bere, T., Dalu, T., and Mwedzi, T. Detecting the impact of heavy metal contaminated sediment on benthic macroinvertebrate communities in tropical streams. Sci. Total Environ. 572<bverticalalign:super;>,</bvertical-align:super;> 147-156. https://doi.org/10.1016/j.scitotenv.2016.07.204 (2016).

91. Lento, J. et al. Diversity of diatoms, benthic macroinvertebrates, and fish varies in response to different environmental correlates in Arctic rivers across North America. Freshw. Biol. 67<bverticalalign:super;>,</bvertical-align:super;> 95-115. https://doi.org/10.1111/fwb.13600 (2022).

92. Ashton, N.N. and Stewart, R.J. Aquatic caddisworm silk is solidified by environmental metal ions during the natural fiber-spinning process. FASEB J. 33<bvertical-align:super;>,</bvertical-align:super;> 572-583. https://doi.org/10.1096/fj.201801029R (2019).

93. Ashton, N.N., Roe, D.R., Weiss, R.B., Cheatham III, T.E. and Stewart, R.J. Self-tensioning aquatic caddisfly silk: $\mathrm{Ca}^{2+}$-dependent structure, strength, and load cycle hysteresis. Biomacromolecules 14<bvertical-align:super;>,</bvertical-align:super;> 3668-3681. https://doi.org/10.1021/bm401036z (2013).

94. Ashton, N.N., Wang, C.S. and Stewart, R.J. The adhesive tape-like silk of aquatic caddisworms. In Biological Adhesives (eds Smith A.) 107-128. https://doi.org/10.1007/978-3-319-46082-6_5 (Springer, 2016).

95. Wilson, K.P. and Blinn, D.W. Food web structure, energetics, and importance of allochthonous carbon in a desert cavernous limnocrene: Devils Hole, Nevada. West. N. Am. Nat. 67<bvertical-align:super;>, </bvertical-align:super;> 185-198. https://doi.org/10.3398/1527-

0904(2007)67[185:FWSEAI]2.0.C0;2 (2007).

96. Tichá, K., Simon, O.P., Douda, K., and Kubikova, L. Detrital components in submontane organogenic springs in relation to their morphology, microhabitats and macroinvertebrates. Pol. J. Ecol. 60<bvertical-align:super;>,</bvertical-align:super;> 163-175. (2012).

97. Marks, J.C. Revisiting the fates of dead leaves that fall into streams. Annu. Rev. Ecol. Evol. 50<bvertical-align:super;>,</bvertical-align:super;> 547-568. https://doi.org/10.1146/annurevecolsys-110218-024755 (2019).

98. Bärlocher, F. and Kendrick, B. Leaf-conditioning by microorganisms. Oecologia $20<$ bverticalalign:super;>,</bvertical-align:super;> 359-362. https://doi.org/10.1007/BF00345526 (1975).

99. Couch, C.A., Meyer, J.L., and Hall Jr, R.O. Incorporation of bacterial extracellular polysaccharide by black fly larvae (Simuliidae). J. North Am. Benthol. Soc. 15<bvertical-align:super;>,</bverticalalign:super;> 289-299. https://doi.org/10.2307/1467277 (1996).

100. Hall Jr, R.O. and Meyer, J.L. The trophic significance of bacteria in a detritus-based stream food web. Ecology <bvertical-align:super;>79</bvertical-align:super;><bvertical-align:super; $></$ bverticalalign:super;><bvertical-align:super;></bvertical-align:super;>1995-2012. https://doi.org/10.1890/0012-9658(1998)079[1995:TTSOBI]2.0.C0;2 (1998).

101. Anderson, T.R., Pond, D.W., and Mayor, D.J. The role of microbes in the nutrition of detritivorous invertebrates: a stoichiometric analysis. Front. Microbiol. 7<bvertical-align:super;>,</bvertical- 
align:super;> 2113. https://doi.org/10.3389/fmicb.2016.02113 (2017).

102. Davis, J.A. and Barmuta, L.A. An ecologically useful classification of mean and near-bed flows in streams and rivers. Freshw. Biol. 21<bvertical-align:super;>,</bvertical-align:super;> 271-282. https://doi.org/10.1111/j.1365-2427.1989.tb01365.x (1989).

103. Battin, T.J., Kaplan, L.A., Newbold, J.D., Cheng, X., and Hansen, C. Effects of current velocity on the nascent architecture of stream microbial biofilms. Appl. Environ. Microbiol. $69<$ bverticalalign:super;>,</bvertical-align:super;> 5443-5452. https://doi.org/10.1128/AEM.69.9.54435452.2003 (2003).

104. Nogaro, G., Datry, T., Mermillod-Blondin, F., Descloux, S., and Montuelle, B. Influence of streambed sediment clogging on microbial processes in the hyporheic zone. Freshw. Biol. 55<bverticalalign:super;>,</bvertical-align:super;> 1288-1302. https://doi.org/10.1111/j.13652427.2009.02352.x (2010).

105. Manga, M. Using springs to study groundwater flow and active geologic processes. Annu. Rev. Earth Planet. Sci. 29<bvertical-align:super;>,</bvertical-align:super;><bvertical-align:super;></bverticalalign:super;>201-228. https://doi.org/10.1146/annurev.earth.29.1.201 (2001).

106. Gascoyne, M. Hydrogeochemistry, groundwater ages and sources of salts in a granitic batholith on the Canadian Shield, southeastern Manitoba. J. Appl. Geochem. 19<bvertical-align:super;>, </bvertical-align:super;> 519-560. https://doi.org/10.1016/S0883-2927(03)00155-0 (2004).

107. Ben Maamar, S. et al. Groundwater isolation governs chemistry and microbial community structure along hydrologic flowpaths. Front. Microbiol. 6:1457. https://doi.org/10.3389/fmicb.2015.01457 (2015).

108. Yan, L. et al. Environmental selection shapes the formation of near-surface groundwater microbiomes. Water Res. 170<bvertical-align:super;>,</bvertical-align:super;> 115341. https://doi.org/10.1016/j.watres.2019.115341 (2020).

\section{Figures}


A

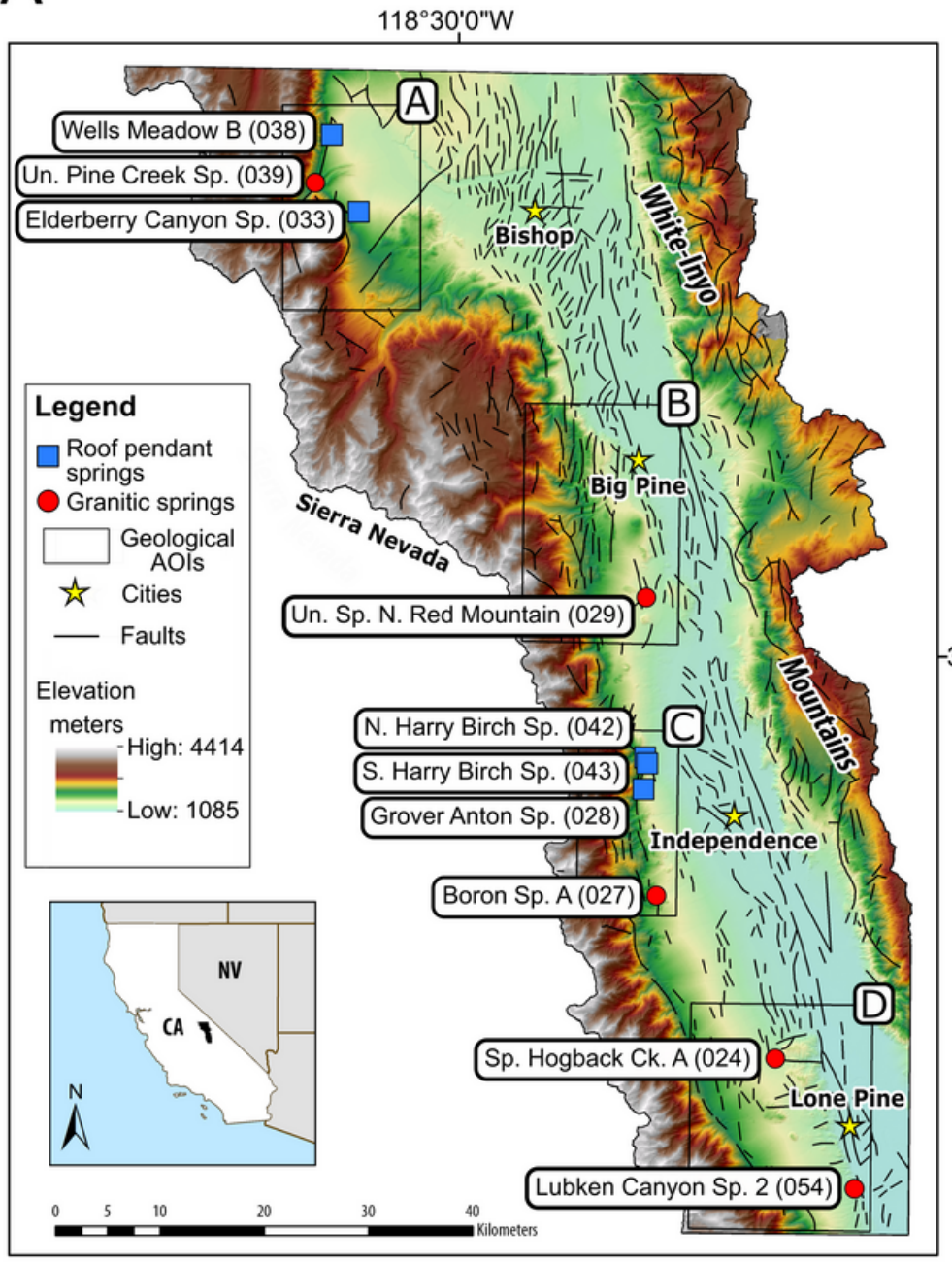

B
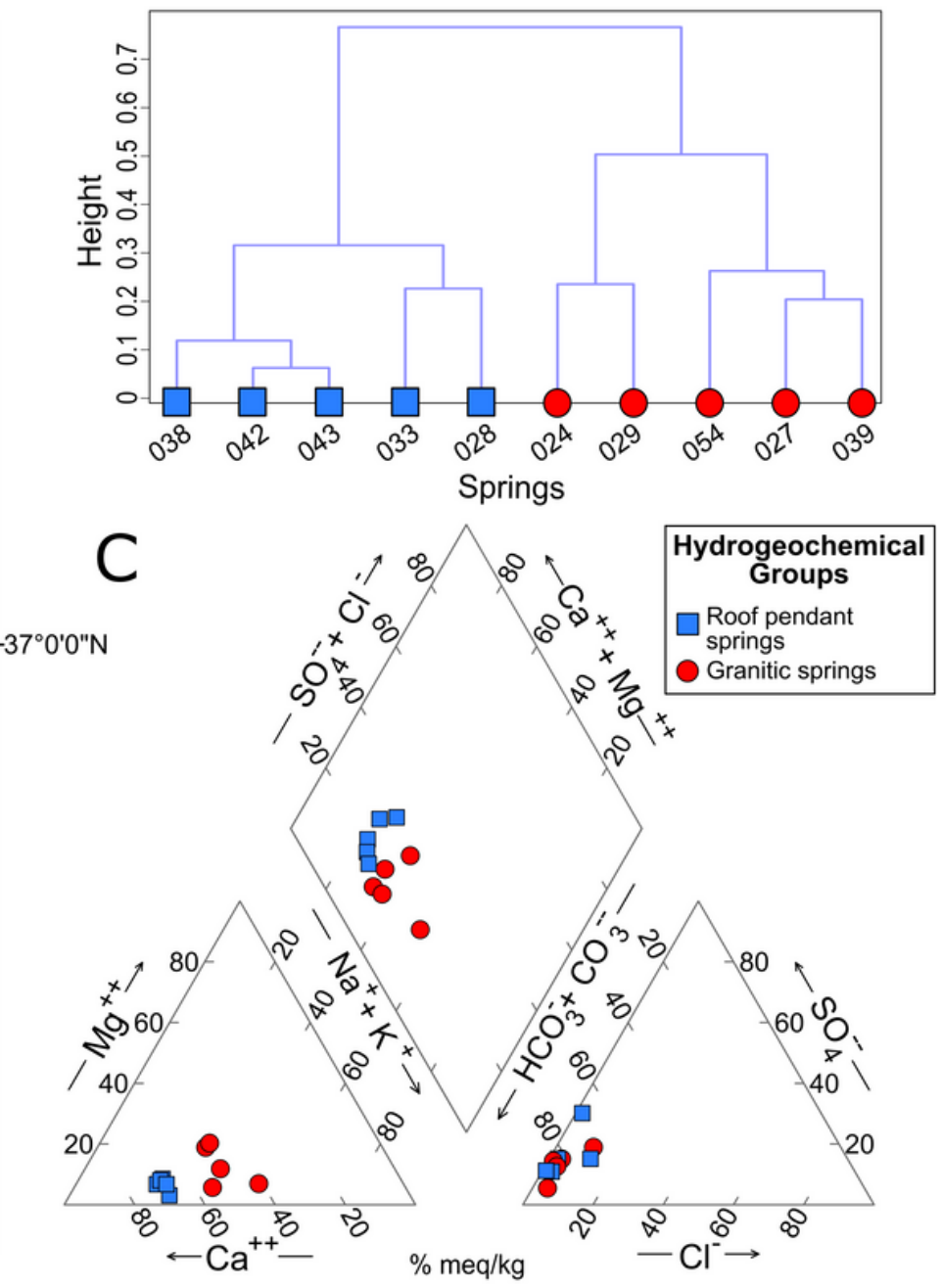

Figure 1

Location and geochemical signatures of two distinct spring groups in Owens Valley. A) Study area map of Owens Valley shaded by elevation (range: 1085-4414 m). Major faults and geological areas of interest (AOIs) are marked on the map with black lines and rectangles. A simplified geological setting map detailing the major geological units and petrologic profiles of the geological AOls is shown in Supplementary Figure 2. B) Dendrogram from the hierarchical clustering analysis (HCA) of the seven major ions in the ten study springs. The HCA was conducted using $\% \mathrm{meq} / \mathrm{kg}$ for each ion. C) Piper diagram displaying geochemical composition of spring waters. 
Benthic microbes
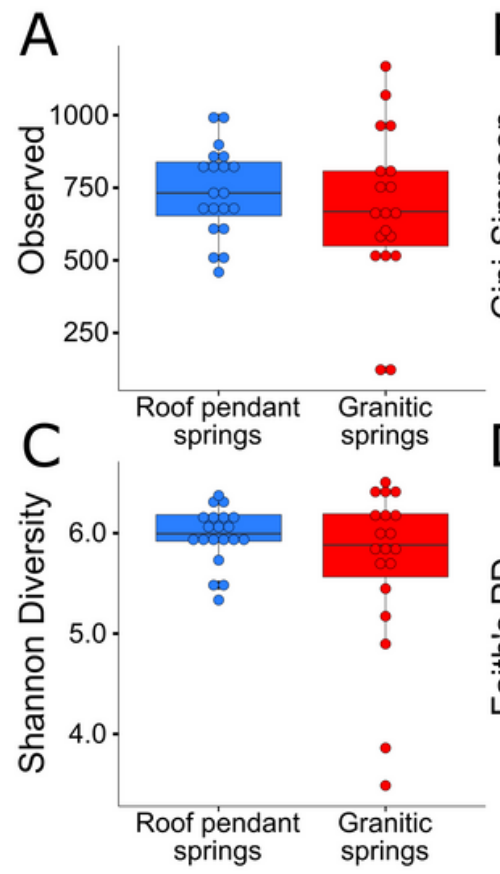

$B_{1.00+}$
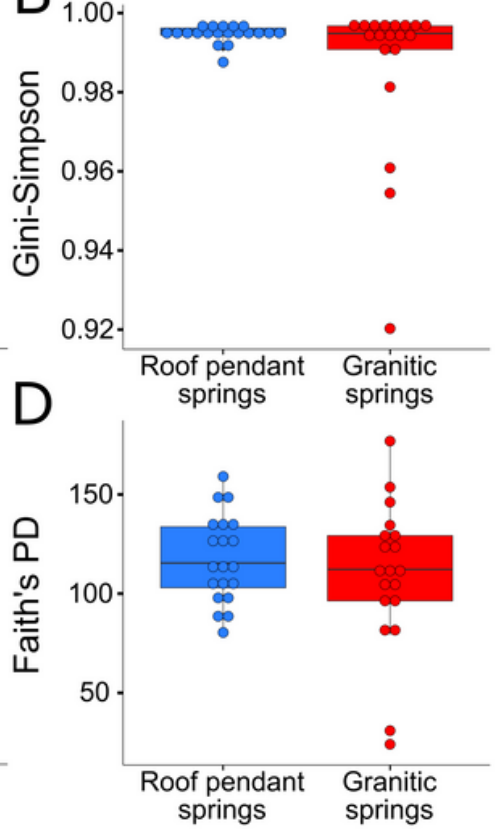

Benthic macroinvertebrates

$E$

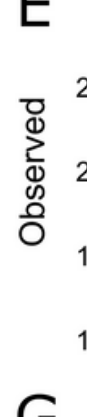

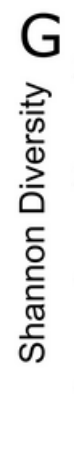

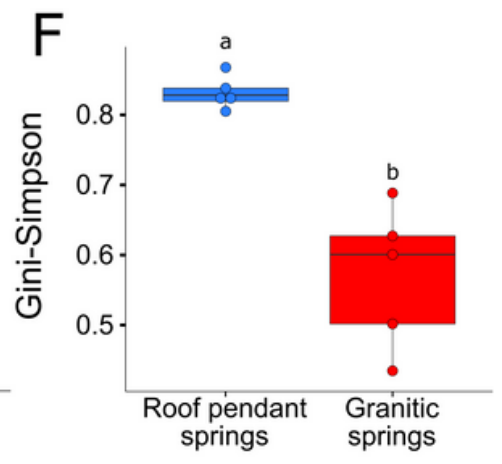

Figure 2

Benthic macroinvertebrate diversity, but not microbial diversity, is higher in roof pendant springs. Boxplots of A) Observed sequence variants, B) Gini-Simpson Index, C) Shannon Diversity Index, and D) Faith's phylogenetic diversity (Faith's PD) values for benthic microbial communities. Boxplots were also made of E) Observed counts, F) Gini-Simpson Index, and G) Shannon Diversity Index for BMI communities. All boxes are colored according to geochemical grouping: roof pendant springs (blue) and granitic springs (red). IQRs, medians, and data points are shown. For the benthic microbial community, no statistically significant differences were observed between the hydrogeochemical groups. For the BMI community, richness and diversity were higher $(p$-value $<0.05)$ in roof pendant springs. 

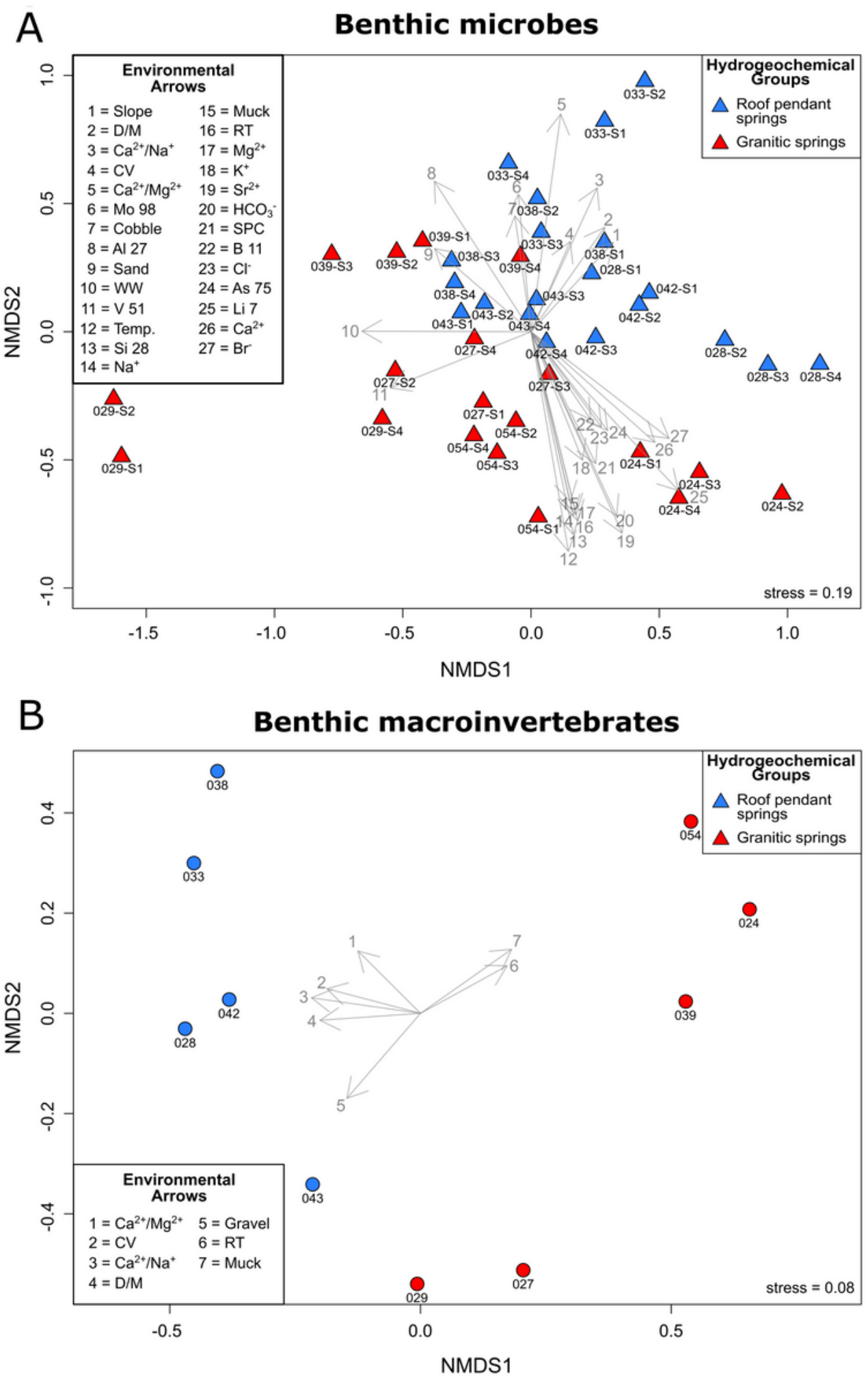

Figure 3

Both microbes and BMls roof pendant spring communities are unique. NMDS ordination of Bray-Curtis dissimilarity between ten springs for both $\mathbf{A}$ ) benthic microbes and $\mathbf{B}$ ) BMls. Each point is colored by geochemical grouping: roof pendant springs (blue) and granitic springs (red). Physicochemical factors deemed to be significantly ( $p$-value $<0.05$ ) correlated with community dissimilarity were overlaid on the NMDS plot. Names of some environmental parameters have been abbreviated on the plot: current 
velocity (CV), wetted width of the spring run (WW), the divalent to monovalent cation ratio (D/M), residence time (RT), and specific conductance (SPC). The influence of hydrogeochemical grouping on $\beta$ diversity was found to be significant via ANOSIM for both benthic microbial communities $(R=0.28, p$ value $=0.001)$ and $\mathrm{BMI}$ communities $(R=0.65$, $p$-value $=0.009)$.

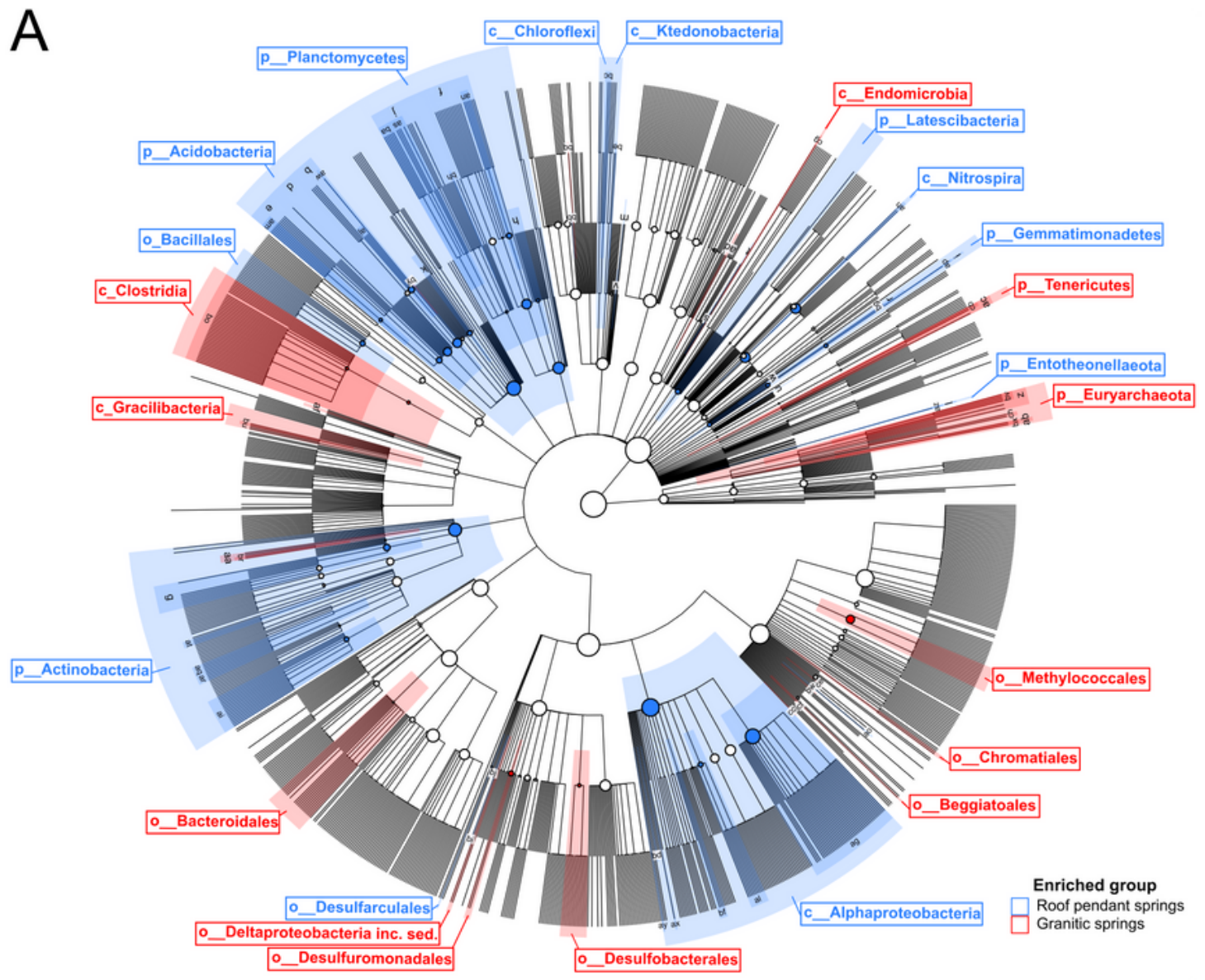

\section{B}

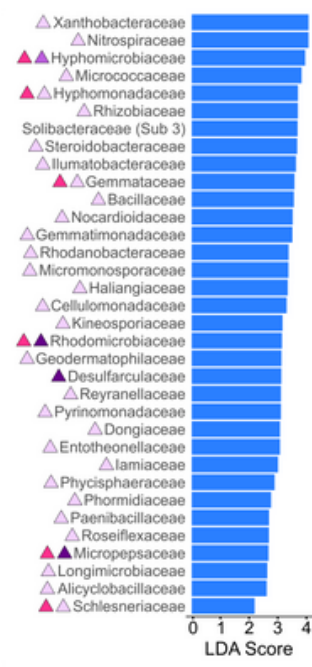

C

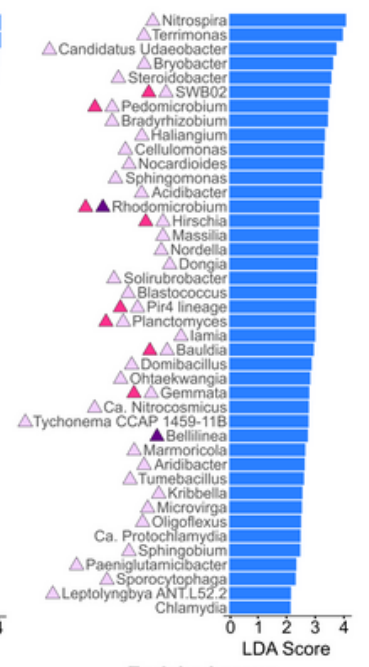

Enriched group

Roof pendant sp

Granitic springs

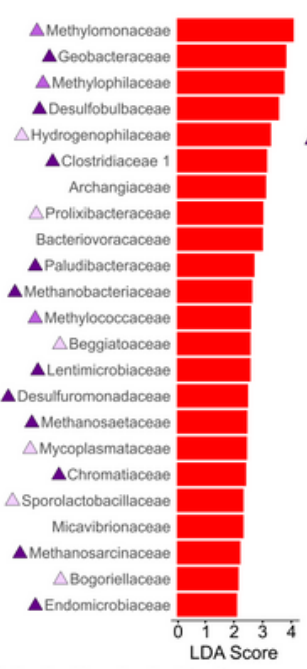

Physiological/ecological traits

Aerobe/facult. anaerobe $\Delta$ Methylotroph

$\Delta$ Prosthecae/stalk

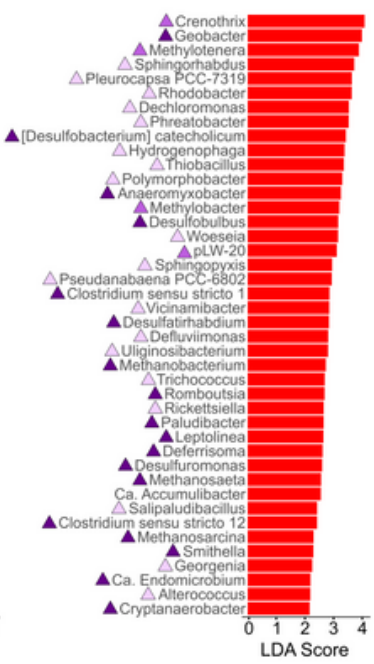

DA Score

Figure 4 


\section{LEfSe analysis reveals physiological differences between microbial communities in the spring groups.}

Linear discriminant analysis (LDA) effect size (LEfSe) A) cladogram and $B+C$ ) barplots depicting microbial taxa enriched ( $p$-value $<0.05$ ) in either $\mathbf{B}$ ) roof pendant springs (blue) or $\mathbf{C}$ ) granitic springs (red). Results shown in the barplots are plotted at the family and genus level. Relevant physiological and ecological traits for families and genera are depicted with colored triangles. Taxa with alpha-numeric names that had no inferred physiology/ecology and unclassified taxa were removed at all taxonomic levels for both the cladogram and barplots; the full list of significantly enriched taxa can be found in the supplement (Supplementary Table 9). 

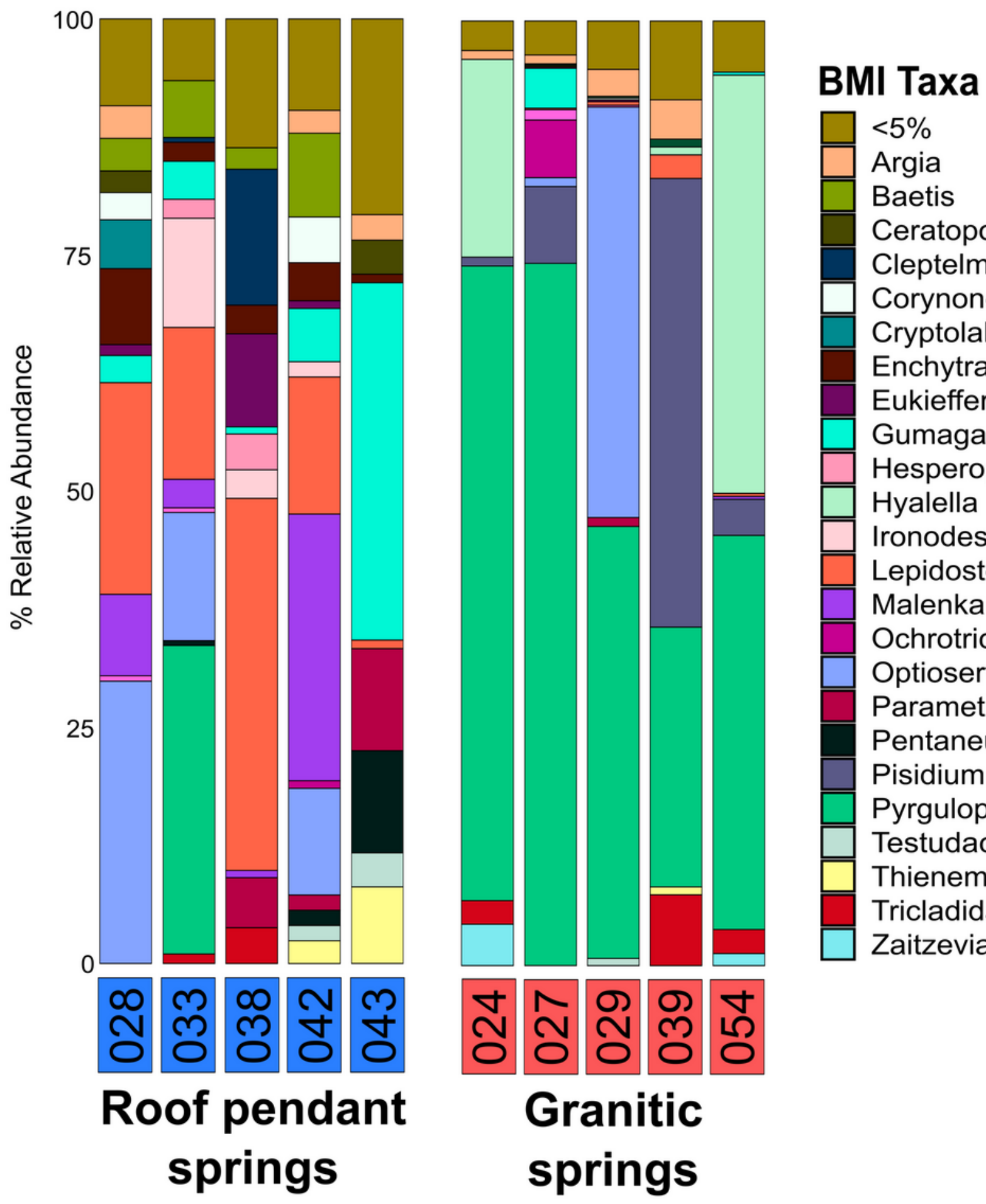

$\square<5 \%$

Baetis

Ceratopogon

Cleptelmis

Corynoneura

Cryptolabis

Enchytraeidae

Eukiefferiella

Gumaga

Hesperoperla

Hyalella

Ironodes

Lepidostoma

Malenka

Ochrotrichia

Optioservus

Parametriocnemus

Pentaneura

Pisidium

Pyrgulopsis

Testudacarus

Thienemannimyia

Tricladida

Zaitzevia

\section{Figure 5}

Benthic macroinvertebrate communities are distinct between geochemical groups. Relative abundance of BMI communities identified microscopically. Roof pendant springs host diverse insect taxa, whereas granitic springs are dominated by Pyrgulopsis. Taxa calculated to be $<5 \%$ across the dataset were agglomerated and plotted as such. Most taxa were identified at the genus level, with the exception of Enchytraeidae (family) and Tricladida (order). 


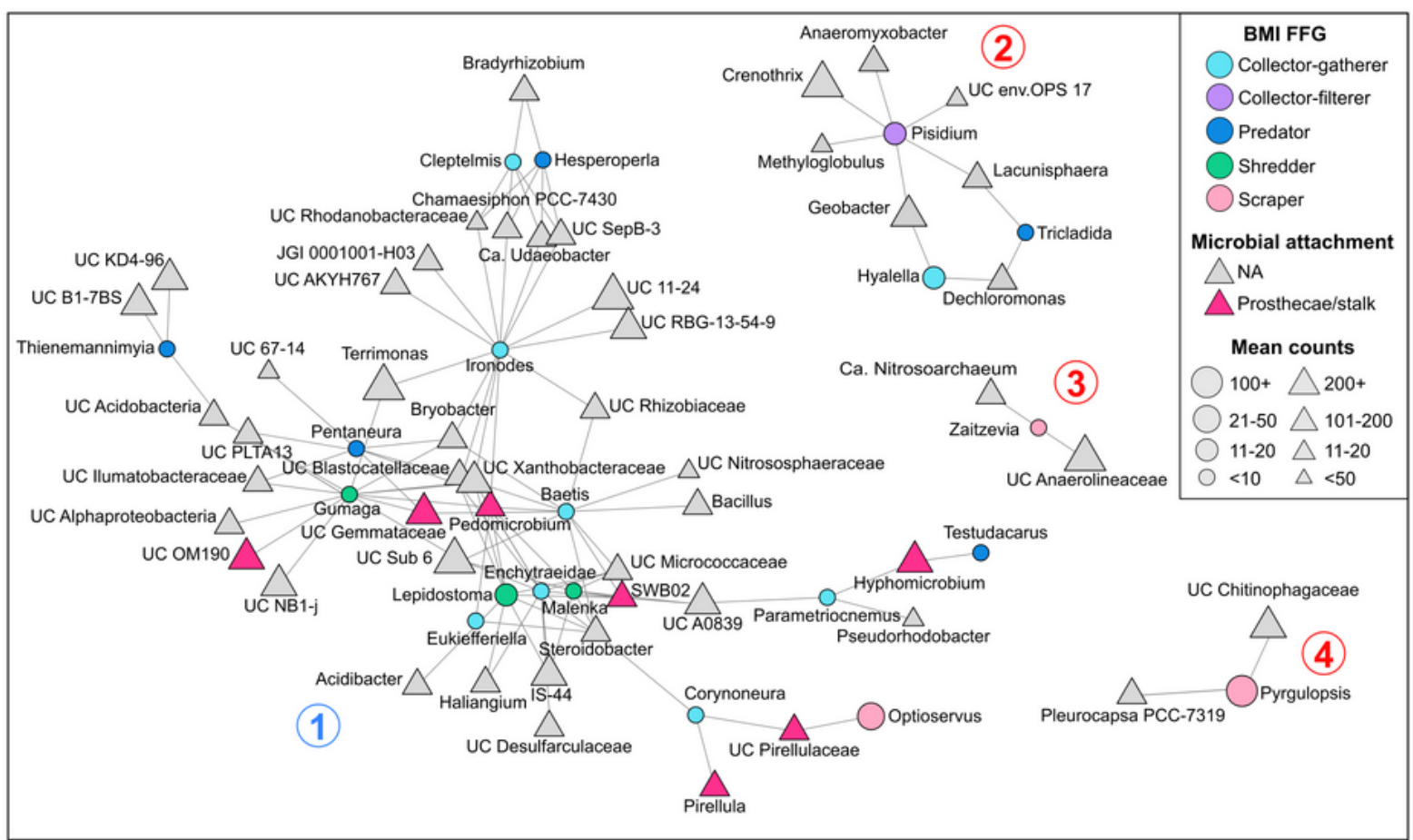

B

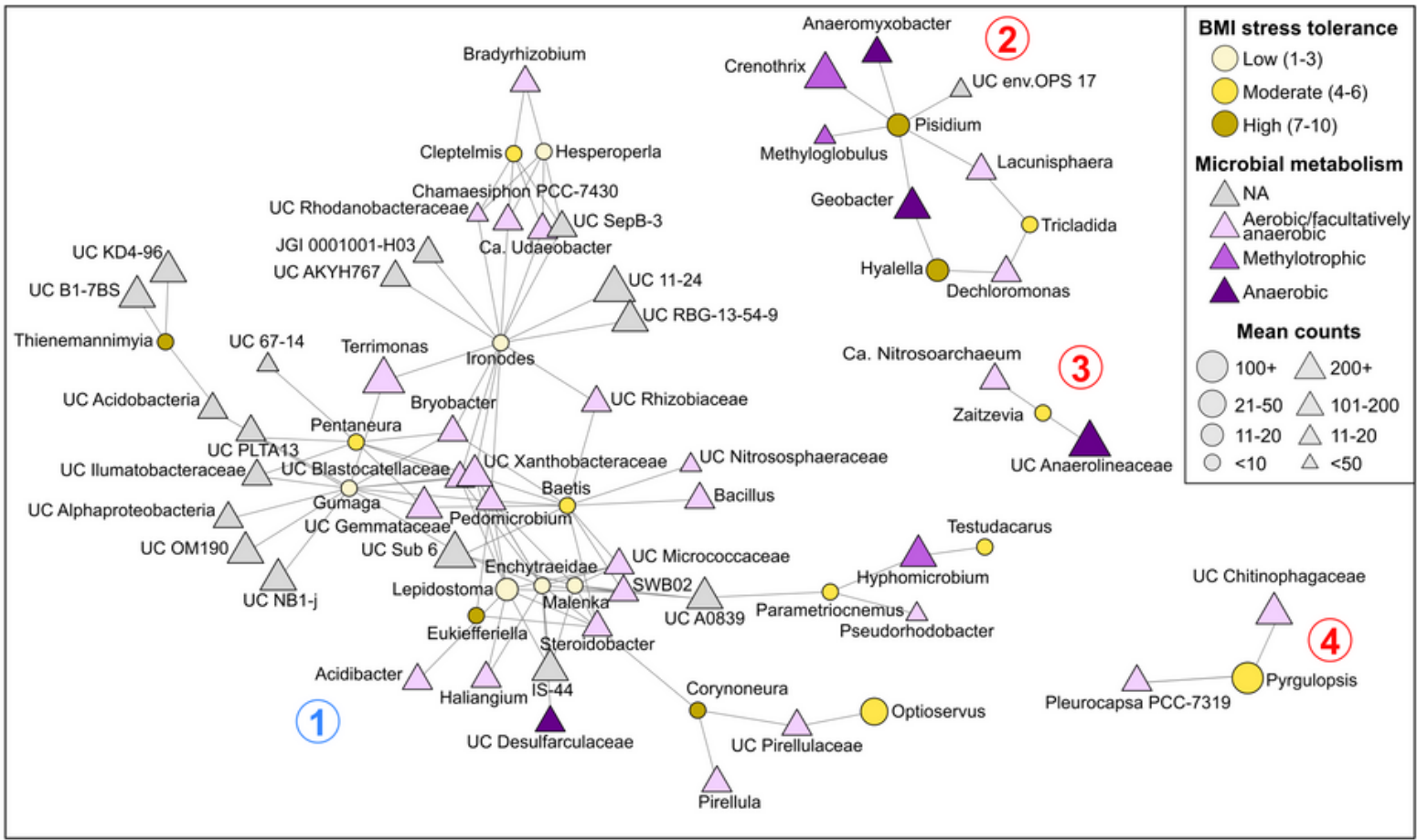

\section{Figure 6}

High connectivity of a BMI-microbe network in roof pendant springs. A co-occurrence network map depicting the positive correlations identified following the Spearman's rank-order correlation analysis performed on relative abundance data from benthic macroinvertebrates (circles) and microbes (triangles) in the ten springs. Nodes are colored by A) BMI functional feeding group (FFG) and microbial attachment or B) BMI stress tolerance and microbial metabolism. Only significant ( $p$-value $<0.05$ ) comparisons and 
nodes with more than one edge are displayed. Major network clusters have been marked with numbers that have been colored to demonstrate the corresponding geochemical grouping.

\section{Supplementary Files}

This is a list of supplementary files associated with this preprint. Click to download.

- FrielRoofPendantSupplementaryTablesv26.xlsx

- FrielRoofPendantSupplementaryFiguresv26.docx 